\title{
THE PARABOLIC-PARABOLIC KELLER-SEGEL MODEL IN $\mathbb{R}^{2 *}$
}

\author{
VINCENT CALVEZ ${ }^{\dagger}$ AND LUCILLA CORRIAS ${ }^{\ddagger}$
}

\begin{abstract}
This paper is devoted mainly to the global existence problem for the two-dimensional parabolic-parabolic Keller-Segel system in the full space. We derive a critical mass threshold below which global existence is ensured. Carefully using energy methods and ad hoc functional inequalities, we improve and extend previous results in this direction. The given threshold is thought to be the optimal criterion, but this question is still open. This global existence result is accompanied by a detailed discussion on the duality between the Onofri and the logarithmic Hardy-Littlewood-Sobolev inequalities that underlie the following approach.
\end{abstract}

Key words. chemotaxis, parabolic system, global weak solutions, energy method, Onofri inequality, Hardy-Littlewood-Sobolev inequality.

AMS subject classifications. 35B60, 35Q80, 92C17, 92B05

\section{Introduction}

Within living organisms, cells may communicate and therefore interact through chemical signals. This signaling pathway is of crucial importance for cell particles to move in the right direction or to organize themselves spatially. Biological challenges involving this phenomenon are numerous. It is known to be so actually in immunology and inflammatory processes, in bacterial growth colonies and at some key stages of embryonic development, for instance. Among mathematical models describing spatial organization of biological population through chemical signals $([27,10,15])$, we highlight the following Patlak-Keller-Segel (PKS) model for chemotaxis ([21, 26]:)

$$
\left\{\begin{array}{l}
\frac{\partial n}{\partial t}=\kappa \Delta n-\chi \nabla \cdot(n \nabla c), \quad t>0, x \in \Omega, \\
\varepsilon \frac{\partial c}{\partial t}=\eta \Delta c+\beta n-\alpha c, \quad t>0, x \in \Omega
\end{array}\right.
$$

where $n$ denotes the density of a cell population and $c$ is the concentration of a chemical signal attracting the cells. The parameters $\kappa, \chi$ and $\beta$ are given positive constants, while $\varepsilon, \eta$ and $\alpha$ are given non-negative constants determining the type of evolution undergone by $c$. The set $\Omega$ is either a bounded domain in $\mathbb{R}^{d}$ or the whole space $\mathbb{R}^{d}$. In any case, boundary conditions or decay conditions at infinity have to be given for the densities $n$ and $c$ together with the initial conditions $n(\cdot, 0)=n_{0}$ and $c(\cdot, 0)=c_{0}$ if $\varepsilon>0$.

The modeling interest of such a system is to exhibit a phenomenon of "critical mass" at least in dimension $d=2$. In a system like (PKS), the coupling between the cell equation and the chemical equation is a positive feedback: the more cells are aggregated, the more they produce a signal attracting other cells. This process is counter-balanced by pure diffusion of the cells, but if the amount of cells is sufficient, this non-local chemical interaction dominates and cells attract themselves.

\footnotetext{
*Received: Noveember 30, 2007; accepted: April 1, 2008. Communicated by Peter Markowich.

${ }^{\dagger}$ Département de Mathématiques et Applications, École Normale Supérieure, CNRS UMR8553, 45 rue d'Ulm, F 75230 Paris cedex 05 (vincent.calvez@ens.fr).

¥Département de Mathématiques, Université d'Evry Val d’Essonne, Rue du Père Jarlan, F 91025 Evry Cedex (lucilla.corrias@univ-evry.fr). The authors warmly thank Benoît Perthame for challenging discussions. The authors acknowledge the support from European Union Marie Curie network M3CS-TuTh, MRTN-CT-2004-503661, ANR project MACBAC.
} 
Thus model (PKS) provides a simple phenomenological description of an instability broadly encountered in biology. The starvation stage of the slime mold amoebae Dictyostelium discoideum for instance is governed by this process, driving the population of unicellular organisms into a multicellular one ([28, 18]). More recently, a (PKS)type model has been suggested to solve a remarkable pattern formation issue in the human brain $[20]$.

It is not overemphasizing to say that system (PKS) has been the subject of a huge quantity of mathematical analysis over the last thirty years. The results of all these investigations can be simply summarized by saying that the global existence or the blow-up of solutions of (PKS) is a space dimension dependent phenomenon. In particular, in dimension $d=2$, the above biological instability has been precisely described mathematically at least when $\varepsilon=\alpha=0$. Indeed, for the parabolic-elliptic system it has been shown that there exists a threshold for the initial mass $M=$ $\int_{\Omega} n_{0}(x) d x$. For values of $M$ under this threshold the solution exists globally in time, while above this threshold the solution blows up in finite time $([2,6],[16,24])$. The modality of the blow-up has also been analyzed [17], as well as the critical case, i.e. when the initial mass $M$ equals the threshold value $([5,3])$.

Let us mention that in dimension $d \geq 3$ a similar critical phenomenon has been investigated. In this case, the $L^{\frac{d}{2}}$-norm of the initial density $n_{0}$ plays the same role as the initial mass $M$ in dimension $d=2$. Indeed, in $[11,12,13]$ the authors proved the global existence of weak solution of system (PKS) of parabolic-parabolic, parabolic-elliptic and parabolic degenerate type under a smallness condition on $\left\|n_{0}\right\|_{L^{d / 2}}$. However, up to our knowledge, blow-up for large $\left\|n_{0}\right\|_{L^{d / 2}}$ is still open, and we conjecture that no critical threshold exists as in dimension 2.

Despite all these results, mathematical open problems around (PKS) still persist, especially for the full parabolic-parabolic system (PKS) $(\varepsilon>0)$. Let us observe that, whenever $\varepsilon=0$ (quasi-stationary hypothesis for the chemical $c$ ), the system reduces to a single parabolic equation with a quadratic nonlocal nonlinearity, $c$ being expressed as a convolution between $n$ and a kernel. On the other hand, when $\varepsilon>0$ the full parabolic-parabolic system is more difficult to handle, since the equations are strongly coupled. However, we will see that in this case the densities $n$ and $c$ play dual roles in some sense (as is highlighted by the dual inequalities used throughout this paper), providing some interesting features and structure to the system (PKS).

The goal of this paper is to tackle the global existence problem for the full parabolic-parabolic system (PKS) with $\varepsilon>0, \eta>0, \alpha \geq 0$ in dimension $d=2$ and in the whole space $\mathbb{R}^{2}$. Indeed, for this problem the optimal threshold of $M$ for the global existence of solutions has not been found yet. A result exists in this direction in [23], but it does not give the exact critical mass. Here we obtain the optimal critical mass value using the energy method $([16,2,6])$ and $a d$ hoc functional inequalities on $\mathbb{R}^{2}$. Once again, the free energy functional

$$
\begin{array}{r}
\mathcal{E}(t)=\int_{\mathbb{R}^{2}} n(x, t) \log n(x, t) d x-\int_{\mathbb{R}^{2}} n(x, t) c(x, t) d x \\
+\frac{1}{2} \int_{\mathbb{R}^{2}}|\nabla c(x, t)|^{2} d x+\frac{\alpha}{2} \int_{\mathbb{R}^{2}} c^{2}(x, t) d x
\end{array}
$$

comes out to be the key ingredient leading to the global existence of solutions under the optimal smallness condition for the mass. Indeed, $\mathcal{E}(t)$ together with its evolution equation provide a gallery of a priori estimates on the solutions $(n, c)$ and we shall make use of each of them. For instance, they allow to prove that the cellular flux 
in $(1.1)$ is $n(\nabla(\log n-c)) \in L^{1}\left(\mathbb{R}_{\text {loc }}^{+} \times \mathbb{R}^{2}\right)$. Therefore, the equation on $n$ holds in the distribution sense. Surprisingly, no specific restrictions on $c_{0}$ are required even for the fully parabolic-parabolic case under consideration, except of course suitable regularity of the initial data.

It is convenient to nondimensionalize system (PKS) through the following change of variables:

$$
t \rightarrow \tau=\kappa t, \quad n(x, t) \rightarrow \tilde{n}(x, \tau)=\frac{\beta \chi}{\eta \kappa} n\left(x, \frac{\tau}{\kappa}\right), \quad c(x, t) \rightarrow \tilde{c}(x, \tau)=\frac{\chi}{\kappa} c\left(x, \frac{\tau}{\kappa}\right) .
$$

Therefore, the system under consideration will be

$$
\left\{\begin{aligned}
\partial_{t} \tilde{n} & =\Delta \tilde{n}-\nabla \cdot(\tilde{n} \nabla \tilde{c}), \quad t>0, x \in \mathbb{R}^{2}, \\
\tilde{\varepsilon} \partial_{t} \tilde{c} & =\Delta \tilde{c}+\tilde{n}-\tilde{\alpha} \tilde{c}, \quad t>0, x \in \mathbb{R}^{2}, \\
\tilde{n}(\cdot, 0) & =\tilde{n}_{0}(\cdot)=\frac{\beta \chi}{\eta \kappa} n_{0}(\cdot), \quad \tilde{c}(\cdot, 0)=\tilde{c}_{0}(\cdot)=\frac{\chi}{\kappa} c_{0}(\cdot), \quad x \in \mathbb{R}^{2} .
\end{aligned}\right.
$$

with $\tilde{\varepsilon}=\frac{\varepsilon \kappa}{\eta}>0$ and $\tilde{\alpha}=\frac{\alpha}{\eta} \geq 0$. The tilde sign will be removed, for clarity. Moreover, fast decay conditions at infinity for $n$ and $c$ have to be associated with (1.1). Concerning the cell density, this decay will be expressed in terms of moments of $n$.

After this change of variables, the only parameters of the system to deal with are the total mass of cells $M=\int_{\mathbb{R}^{2}} n_{0}(x) d x$, which is conserved over time, the inverse diffusion rate of the chemical $\varepsilon$ and the chemical degradation rate $\alpha \geq 0$. The latter seems to play no essential role, unless it induces slightly technical difficulties in the estimates.

Our main results are the followings.

Theorem 1.1 (Global existence). Assume $\varepsilon>0$ and $\alpha \geq 0$. Let $\left(n_{0}, c_{0}\right)$ be nonnegative initial conditions for the parabolic-parabolic system (1.1) such that

(H1) $n_{0} \in L^{1}\left(\mathbb{R}^{2}\right) \cap L^{1}\left(\mathbb{R}^{2}, \log \left(1+|x|^{2}\right) d x\right)$ and $n_{0} \log n_{0} \in L^{1}\left(\mathbb{R}^{2}\right)$;

(H2) $c_{0} \in H^{1}\left(\mathbb{R}^{2}\right)$ if $\alpha>0$ while $c_{0} \in L^{1}\left(\mathbb{R}^{2}\right)$ and $\left|\nabla c_{0}\right| \in L^{2}\left(\mathbb{R}^{2}\right)$ if $\alpha=0$;

(H3) $n_{0} c_{0} \in L^{1}\left(\mathbb{R}^{2}\right)$.

Assume in addition that the mass is sub-critical, i.e., $M<8 \pi$. Then there exists a global weak non-negative solution $(n, c)$ of (1.1) such that

$$
\begin{gathered}
n \in L^{\infty}\left((0, \infty) ; L^{1}\left(\mathbb{R}^{2}\right)\right) \cap L_{l o c}^{\infty}\left((0, \infty) ; L^{1}\left(\mathbb{R}^{2}, \log \left(1+|x|^{2}\right) d x\right)\right) \\
\text { and } n \log n \in L_{l o c}^{\infty}\left((0, \infty) ; L^{1}\left(\mathbb{R}^{2}\right)\right) ; \\
c \in L_{l o c}^{\infty}\left((0, \infty) ; H^{1}\left(\mathbb{R}^{2}\right)\right) \quad \text { if } \alpha>0 ; \\
c \in L_{l o c}^{\infty}\left((0, \infty) ; L^{1}\left(\mathbb{R}^{2}\right)\right) \quad \text { and } \quad|\nabla c| \in L_{l o c}^{\infty}\left((0, \infty) ; L^{2}\left(\mathbb{R}^{2}\right)\right) \quad \text { if } \alpha=0 ; \\
\partial_{t} c \in L_{l o c}^{2}\left((0, \infty) ; L^{2}\left(\mathbb{R}^{2}\right)\right) \quad \text { and } \quad n c \in L_{l o c}^{\infty}\left((0, \infty) ; L^{1}\left(\mathbb{R}^{2}\right)\right) ; \\
\mathcal{E}(t)+\int_{0}^{t} \int_{\mathbb{R}^{2}} n(x, s)|\nabla(\log n(x, s)-c(x, s))|^{2} d x d s+\varepsilon \int_{0}^{t} \int_{\mathbb{R}^{2}}\left|\partial_{t} c(x, s)\right|^{2} d x d s \leq \mathcal{E}(0) .
\end{gathered}
$$


Moreover, $n \in L_{\text {loc }}^{\infty}\left((0, \infty) ; L^{p}\left(\mathbb{R}^{2}\right)\right)$ for any $1<p<\infty$ (regularizing effect).

Let us observe that the global existence result given in Theorem 1.1 holds true under the optimal condition $n_{0} \in L^{1}\left(\mathbb{R}^{2}, \log \left(1+|x|^{2}\right) d x\right)$. This is weaker than the hypothesis $n_{0} \in L^{1}\left(\mathbb{R}^{2},|x|^{2} d x\right)$ used in [6] for the global existence result in the case $\varepsilon=$ $\alpha=0$. As a matter of fact, we can adapt Theorem 1.1 to the case $\varepsilon=\alpha=0$ and slightly improve the result in [6], (see Appendix A.3). On the other hand, the hypothesis $n_{0} \in L^{1}\left(\mathbb{R}^{2},|x|^{2} d x\right)$ remains still necessary to obtain a blow-up result for the parabolicelliptic system (1.1). Nevertheless, we can extend the blow-up result in [6] to the case $\alpha \geq 0$ as follows. The same blow-up result for the full parabolic-parabolic system is still, to our knowledge, an open problem.

Theorem 1.2 (Blow-up). Assume $\varepsilon=0$ and $\alpha \geq 0$. Let $n_{0}$ be a non-negative $L^{1}\left(\mathbb{R}^{2}\right)$ function with super-critical mass, i.e. $M>8 \pi$ and finite second momentum $I_{0}$. Let $n$ be a non-negative smooth solution of (1.1) with $c=B_{\alpha} * n$ and $B_{\alpha}$ the Bessel kernel, and let $\left[0, T^{*}\right)$ be the maximal interval of existence. Then, there exists a positive universal constant $\mathcal{C}$ such that if

$$
\alpha \int_{\mathbb{R}^{2}}|x|^{2} n_{0}(x) d x \leq \frac{1}{4 \mathcal{C}^{2} M}(M-8 \pi)^{2},
$$

the solution blows up and $T^{*} \leq \frac{2 \pi I_{0}}{M\left(M-8 \pi-2 \mathcal{C} \sqrt{\alpha M I_{0}}\right)}$.

The paper is organized as follows. In Section 2 we give a set of technical tools to be used in the sequel and we describe briefly the dual strategies that give the required a priori estimates. In Section 3 we derive the a priori estimates both in cases $\alpha>0$ and $\alpha=0$ (among which is the key equi-integrability estimate) from the so-called Onofri's inequality on the whole space $\mathbb{R}^{2}$. In Section 4 we re-derive those estimates using the dual strategy based on the logarithmic Hardy-Littlewood-Sobolev inequality. Section 5 is devoted to the proof of the regularizing effect acting on the solutions. Section 6 is a short description of the regularization procedure which leads to the rigorous proof of global existence when combined with the a priori estimates derived in Sections 3 and 4. Blow-up of the solutions in the special case $\varepsilon=0$ is shown for a super-critical mass in Section 7, under a smallness condition on $\alpha \int_{\mathbb{R}^{2}}|x|^{2} n_{0}(x) d x$. Finally, several complementary results are given for the sake of completeness in Section A.

In the sequel, we will denote by $C$ every positive constant that may vary from line to line in the computations. Only the dependence on crucial parameters will be written explicitly. Moreover, $(u)_{+}$and $(u)_{-}$will denote the positive and negative part of $u$ as usual, while $L_{+}^{1}\left(\mathbb{R}^{2}\right):=\left\{f \in L^{1}\left(\mathbb{R}^{2}\right), f \geq 0\right\}$.

\section{The free energy and the moments control}

It is well known that system (1.1) is equipped with the following free energy functional:

$$
\begin{array}{r}
\mathcal{E}(t)=\int_{\mathbb{R}^{2}} n(x, t) \log n(x, t) d x-\int_{\mathbb{R}^{2}} n(x, t) c(x, t) d x \\
+\frac{1}{2} \int_{\mathbb{R}^{2}}|\nabla c(x, t)|^{2} d x+\frac{\alpha}{2} \int_{\mathbb{R}^{2}} c^{2}(x, t) d x .
\end{array}
$$

In the kinetic equation literature, the first term $\int_{\mathbb{R}^{2}} n(x, t) \log n(x, t) d x$ is usually referred to as the physical entropy. However, here it will be more convenient and natural to define the entropy in the line of [9] as

$$
E(n ; c)(t)=\int_{\mathbb{R}^{2}}(n(x, t) \log n(x, t)-n(x, t) c(x, t)) d x,
$$


including also the potential energy term $\int_{\mathbb{R}^{2}} n(x, t) c(x, t) d x$. On the other hand, the potential energy term has also to be included in the chemical energy associated to the elliptic equation $-\Delta c+\alpha c=n$, i.e.

$$
F_{\alpha}(c ; n)(t)=\frac{1}{2} \int_{\mathbb{R}^{2}}|\nabla c(x, t)|^{2} d x+\frac{\alpha}{2} \int_{\mathbb{R}^{2}} c^{2}(x, t) d x-\int_{\mathbb{R}^{2}} n(x, t) c(x, t) d x, \quad \alpha \geq 0 .
$$

Thereby, the free energy $\mathcal{E}(t)$ is a superposition of (2.2) and (2.3), thus reflecting the strongly coupled property of system (1.1). The quantity $\mathcal{E}(t)$ will play a fondamental role in the research of a priori estimates starting from the following proposition.

Proposition 2.1. Let $(n, c)$ be any non-negative and sufficiently smooth solution of (1.1) with finite free energy (2.1). Then $\mathcal{E}(t)$ decreases along the trajectories of the dynamical system associated to (1.1), since

$$
\frac{d}{d t} \mathcal{E}(t)=-\int_{\mathbb{R}^{2}} n(x, t)|\nabla(\log n(x, t)-c(x, t))|^{2} d x-\varepsilon \int_{\mathbb{R}^{2}}\left|\partial_{t} c(x, t)\right|^{2} d x \leq 0 .
$$

Proof. The equation in $n$ can be written as $\partial_{t} n=\nabla \cdot(n \nabla(\log n-c))$. Then, using mass conservation, we obtain

$$
\begin{array}{r}
\int_{\mathbb{R}^{2}} \partial_{t} n(x, t)(\log n(x, t)-c(x, t)) d x=\frac{d}{d t} \int_{\mathbb{R}^{2}} n(x, t) \log n(x, t) d x \\
-\int_{\mathbb{R}^{2}} \partial_{t} n(x, t) c(x, t) d x \\
-\int_{\mathbb{R}^{2}} n(x, t)|\nabla(\log n(x, t)-c(x, t))|^{2} d x .
\end{array}
$$

On the other hand, testing the equation in $c$ against $\partial_{t} c$, we have

$$
\begin{array}{r}
\varepsilon \int_{\mathbb{R}^{2}}\left|\partial_{t} c(x, t)\right|^{2} d x=-\frac{d}{d t} \int_{\mathbb{R}^{2}} \frac{|\nabla c(x, t)|^{2}}{2} d x+ \\
\int_{\mathbb{R}^{2}} n(x, t) \partial_{t} c(x, t) d x \\
-\alpha \frac{d}{d t} \int_{\mathbb{R}^{2}} \frac{c^{2}(x, t)}{2} d x .
\end{array}
$$

We conclude by summing (2.5) and (2.6).

Equation (2.4) measures the dissipation of the free energy due to the entropy production term

$$
I(t)=\int_{\mathbb{R}^{2}} n(x, t)|\nabla(\log n(x, t)-c(x, t))|^{2} d x
$$

and to the chemical production term $\varepsilon \int_{\mathbb{R}^{2}}\left|\partial_{t} c(x, t)\right|^{2} d x$. Let us observe, however, that any weak solution of (1.1) is not expected to satisfy (2.4) but the inequality

$$
\mathcal{E}(t)+\int_{0}^{t} \int_{\mathbb{R}^{2}} n(x, s)|\nabla(\log n(x, s)-c(x, s))|^{2} d x d s+\varepsilon \int_{0}^{t} \int_{\mathbb{R}^{2}}\left|\partial_{t} c(x, s)\right|^{2} d x d s \leq \mathcal{E}(0),
$$

as under the quasi-stationary hypothesis $\varepsilon=\alpha=0$ (see [6]).

The time-monotonicity of $\mathcal{E}(t)$ given by (2.4) provides us with an upper control of the entropy (2.2). But a control from below of the entropy is also needed in order 
to obtain a priori estimates on the solution and then the global existence result. In the case of the parabolic-parabolic system (1.1) on a bounded domain $\Omega \subset \mathbb{R}^{2}$, the strategy usually followed uses essentially two primary tools: a minimization principle with respect to $n$ of the entropy $E(n ; c)$ and the Moser-Trudinger inequality. Moreover, their combination gives the a priori estimates under the exact critical mass value for $M($ see $[2,7],[16,24])$.

Concerning system (1.1) in the whole space $\mathbb{R}^{2}$ under interest, the first result we give here is that the same method above can be followed. However, in order to do so, one has firstly to reinforce the space decay of $c$ as $|x| \rightarrow+\infty$ in order to minimize the entropy $E(n ; c)$ with respect to $n$ and secondly to employ an ad hoc Moser-Trudinger type inequality, i.e. Onofri's inequality [25].

The second result is that an alternative strategy, dual in some sense to the previous one, can be also adopted. The technical tools to be employed are: the minimization with respect to $c$ of the chemical energy $F_{\alpha}(c ; n)(2.3)$ and the logarithmic HardyLittlewood-Sobolev inequality (HLS in the sequel, see Lemma 4.1 and [8]) if $\alpha=0$, or a modified version of this inequality if $\alpha>0$ (see Lemma 4.2). This method is new, and it is somewhat the extension to the parabolic-parabolic system (1.1) of what was done in [6] for the parabolic-elliptic system (1.1) with $\varepsilon=\alpha=0$. Indeed, in this case the free energy reads as

$$
\mathcal{E}(t)=\int_{\mathbb{R}^{2}} n(x, t) \log n(x, t) d x-\frac{1}{2} \int_{\mathbb{R}^{2}} n(x, t) c(x, t) d x,
$$

with the concentration of the chemical given by $c(x, t)=-\frac{1}{2 \pi} \int_{\mathbb{R}^{2}} \log |x-y| n(y, t) d y$, and hence it is well adapted for applying the HLS inequality.

No matter the method followed to obtain the necessary a priori estimates, we are in any case lead to consider the following modified free energy functional:

$$
\begin{aligned}
\mathcal{E}_{H}(t) & =\mathcal{E}(t)-\int_{\mathbb{R}^{2}} n(x, t) \log H(x) d x \\
& =E(n ; c+\log H)+\frac{1}{2} \int_{\mathbb{R}^{2}}|\nabla c(x, t)|^{2} d x+\frac{\alpha}{2} \int_{\mathbb{R}^{2}} c^{2}(x, t) d x,
\end{aligned}
$$

where

$$
H(x)=\frac{1}{\pi} \frac{1}{\left(1+|x|^{2}\right)^{2}}
$$

has been chosen so that $\mathcal{J}_{\mathcal{S}}:=4 \pi H$ is the Jacobian of the usual stereographic projection on the sphere $\mathcal{S}: \mathbb{R}^{2} \cup\{\infty\} \rightarrow \mathbb{S}^{2}$ and $\int_{\mathbb{R}^{2}} H(x) d x=1$, (see [22]).

The introduction of the function $H$ will appear to the reader more natural in Sections 3 and 4 where the two methods will be developed respectively. Here, let us observe that, by opposition to $\mathcal{E}(t)$, the functional $\mathcal{E}_{H}(t)$ is not time decreasing. However, we can control its time-growth by the following computation. We have

$$
\begin{aligned}
\frac{d}{d t} \int_{\mathbb{R}^{2}} n(x, t) \log H(x) d x & =-\int_{\mathbb{R}^{2}} n(x, t) \nabla \log H(x) \cdot \nabla(\log n(x, t)-c(x, t)) d x \\
& =2 \int_{\mathbb{R}^{2}} n(x, t) \nabla \log \left(1+|x|^{2}\right) \cdot \nabla(\log n(x, t)-c(x, t)) d x
\end{aligned}
$$


and using equation (2.4), we obtain

$$
\begin{aligned}
\frac{d}{d t} \mathcal{E}_{H}(t)= & -\int_{\mathbb{R}^{2}} n(x, t)|\nabla(\log n(x, t)-c(x, t))|^{2} d x-\varepsilon \int_{\mathbb{R}^{2}}\left|\partial_{t} c(x, t)\right|^{2} d x \\
& -2 \int_{\mathbb{R}^{2}} n(x, t) \nabla \log \left(1+|x|^{2}\right) \cdot \nabla(\log n(x, t)-c(x, t)) \\
= & -\int_{\mathbb{R}^{2}} n(x, t)\left|\nabla\left(\log n(x, t)-c(x, t)+\log \left(1+|x|^{2}\right)\right)\right|^{2} d x-\varepsilon \int_{\mathbb{R}^{2}}\left|\partial_{t} c(x, t)\right|^{2} d x \\
& \left.+\int_{\mathbb{R}^{2}} n(x, t) \mid \nabla \log \left(1+|x|^{2}\right)\right)\left.\right|^{2} d x,
\end{aligned}
$$

where

$$
\int_{\mathbb{R}^{2}} n(x, t)\left|\nabla \log \left(1+|x|^{2}\right)\right|^{2} d x=\int_{\mathbb{R}^{2}} \frac{4|x|^{2}}{\left(1+|x|^{2}\right)^{2}} n(x, t) d x \leq M .
$$

As a consequence, $\mathcal{E}_{H}(t)$ grows at most linearly in time.

Before concluding this preliminary section, we state the minimization lemmas for the entropy and the chemical energy respectively. Moreover, we derive also some bounds for the moments of the density $n$ in term of the entropy production (2.7) that is shown to be locally integrable in time in the sequel (see Theorem 3.2).

Lemma 2.1 (The entropy minimization). Let $\psi$ be any function such that $e^{\psi} \in$ $L^{1}\left(\mathbb{R}^{2}\right)$ and denote $\bar{n}=M e^{\psi}\left(\int_{\mathbb{R}^{2}} e^{\psi} d x\right)^{-1}$, with $M$ a positive arbitrary constant. Let $E: L_{+}^{1}\left(\mathbb{R}^{2}\right) \rightarrow \mathbb{R} \cup\{\infty\}$ be the entropy functional

$$
E(n ; \psi)=\int_{\mathbb{R}^{2}}(n(x) \log n(x)-n(x) \psi(x)) d x
$$

and let $R E: L_{+}^{1}\left(\mathbb{R}^{2}\right) \rightarrow \mathbb{R} \cup\{\infty\}$ defined by

$$
R E(n \mid \bar{n})=\int_{\mathbb{R}^{2}} n(x) \log (n(x) / \bar{n}(x)) d x
$$

be the relative (to $\bar{n})$ entropy. Then $E(n ; \psi)$ and $R E(n \mid \bar{n})$ are finite or infinite in the same time and for all $n$ in the set $\mathcal{U}=\left\{n \in L_{+}^{1}\left(\mathbb{R}^{2}\right), \int_{\mathbb{R}^{2}} n(x) d x=M\right\}$ and it holds true that

$$
E(n ; \psi)-E(\bar{n} ; \psi)=R E(n \mid \bar{n}) \geq 0
$$

The entropy minimization Lemma 2.1 is now a classical lemma, and the proof can be found for example in [9], where a more general class of entropy functionals including $E(n ; \psi)$ is considered. Anyway, this lemma being of primary importance and for the sake of completeness, we will give the proof in Appendix A.1.

Lemma 2.2 (The chemical energy minimization). Assume $\alpha \geq 0$, and let $f$ be an $L^{1}\left(\mathbb{R}^{2}\right)$ function such that

(A1) if $\alpha>0$ then $f \geq 0$ and $f \log f \in L^{1}\left(\mathbb{R}^{2}\right)$; 
(A2) if $\alpha=0$ then $f \in L^{1}\left(\mathbb{R}^{2}, \log \left(1+|x|^{2}\right) d x\right), \int_{\mathbb{R}^{2}} f(x) d x=0$ and $f$ can be decomposed as $f=f_{1}+f_{2}$ with $f_{1} \geq 0, f_{1} \in L_{l o c}^{1}\left(\mathbb{R}^{2}\right), f_{1} \log f_{1} \in L^{1}\left(\mathbb{R}^{2}\right)$ and $f_{2} \in$ $L^{2}\left(\mathbb{R}^{2}\right)$.

Finally, let us denote

$$
\bar{c}(x)=\left\{\begin{array}{lll}
\left(B_{\alpha} * f\right)(x) & \text { if } \quad \alpha>0, \\
\left(E_{2} * f\right)(x) & \text { if } \quad \alpha=0,
\end{array}\right.
$$

where $*$ denotes space convolution, $B_{\alpha}$ denotes the Bessel kernel $B_{\alpha}(z)=$ $\frac{1}{4 \pi} \int_{0}^{+\infty} \frac{1}{t} e^{-\frac{|z|^{2}}{4 t}-\alpha t} d t$ and $E_{2}(z)=-\frac{1}{2 \pi} \log |z|$ is the fundamental solution of the Laplace's equation in $\mathbb{R}^{2}$. Then, $\bar{c} \in H^{1}\left(\mathbb{R}^{2}\right)$ when $\alpha>0$, while $|\nabla \bar{c}| \in L^{2}\left(\mathbb{R}^{2}\right)$ when $\alpha=0$, with

$$
\nabla \bar{c}(x)= \begin{cases}\left(\nabla B_{\alpha} * f\right)(x) & \text { if } \alpha>0, \\ \left(\nabla E_{2} * f\right)(x) & \text { if } \alpha=0 .\end{cases}
$$

Moreover, if $\alpha>0$ then for all $c \in H^{1}\left(\mathbb{R}^{2}\right)$, the chemical energy $F_{\alpha}(c ; f)$ is finite and satisfies

$$
F_{\alpha}(c ; f)-F_{\alpha}(\bar{c} ; f)=\frac{1}{2} \int_{\mathbb{R}^{2}}|\nabla(c-\bar{c})(x)|^{2} d x+\frac{\alpha}{2} \int_{\mathbb{R}^{2}}(c-\bar{c})^{2}(x) d x \geq 0,
$$

while if $\alpha=0$ then for all $c$ such that $|\nabla c| \in L^{2}\left(\mathbb{R}^{2}\right)$, the chemical energy $F_{0}(c ; f)$ is finite and satisfies

$$
F_{0}(c ; f)-F_{0}(\bar{c} ; f)=\frac{1}{2} \int_{\mathbb{R}^{2}}|\nabla(c-\bar{c})(x)|^{2} d x \geq 0 .
$$

Let us observe that whenever $f \in L^{2}\left(\mathbb{R}^{2}\right)$ in $(2.12)$ the chemical energy minimization Lemma 2.2 can be obtained easily by applying the variational method, at least for $\alpha>0$. However, we want to use here minimal hypotheses on $f$, and therefore the proof becomes a little more technical, expecially when $\alpha=0$. Again, for the sake of completeness, the proof is given in Appendix A.2.

Lemma 2.3 (Moment Lemma). Let $c$ be a given smooth function. Let $n$ be any non-negative and sufficiently smooth solution of $\partial_{t} n=\Delta n-\nabla \cdot(n \nabla c)$, with fast decay at infinity and total mass $M$. Then, for any $\delta>0$, we have the following bounds for the evolution of the moments of $n$ :

$$
\begin{gathered}
\int_{\mathbb{R}^{2}} n(x, t) \log \left(1+|x|^{2}\right) d x \leq \int_{\mathbb{R}^{2}} n_{0}(x) \log \left(1+|x|^{2}\right) d x+\frac{M}{2 \delta} t \\
+\frac{\delta}{2} \int_{0}^{t} \int_{\mathbb{R}^{2}} n(x, s)|\nabla(\log n(x, s)-c(x, s))|^{2} d x d s \\
\int_{\mathbb{R}^{2}}|x| n(x, t) d x \leq \int_{\mathbb{R}^{2}}|x| n_{0}(x) d x+\frac{M}{2 \delta} t \\
+\frac{\delta}{2} \int_{0}^{t} \int_{\mathbb{R}^{2}} n(x, s)|\nabla(\log n(x, s)-c(x, s))|^{2} d x d s ; \\
\int_{\mathbb{R}^{2}}|x|^{2} n(x, t) d x \leq 2 \int_{\mathbb{R}^{2}}|x|^{2} n_{0}(x) d x+2 t \int_{0}^{t} \int_{\mathbb{R}^{2}} n(x, s)|\nabla(\log n(x, s)-c(x, s))|^{2} d x d s
\end{gathered}
$$


Proof. Writing the equation in $n$ as $\partial_{t} n=\nabla \cdot(n \nabla(\log n-c))$, it follows that

$$
\begin{aligned}
& \frac{d}{d t} \int_{\mathbb{R}^{2}} n(x, t) \phi(x) d x=-\int_{\mathbb{R}^{2}} n(x, t) \nabla \phi(x) \cdot \nabla(\log n(x, t)-c(x, t)) d x \\
\leq & \frac{1}{2 \delta} \int_{\mathbb{R}^{2}}|\nabla \phi(x)|^{2} n(x, t) d x+\frac{\delta}{2} \int_{\mathbb{R}^{2}} n(x, t)|\nabla(\log n(x, t)-c(x, t))|^{2} d x,
\end{aligned}
$$

with $\delta>0$ arbitrary. Taking successively $\phi(x)=\log \left(1+|x|^{2}\right)$ and $\phi(x)=|x|$, observing that with the first choice of $\phi$ we have

$$
|\nabla \phi(x)|=\left|\frac{2 x}{1+|x|^{2}}\right| \leq 1,
$$

and using the mass conservation property, we proved inequalities (2.16) and (2.17). Inequality (2.18) follows in a similar way, since for $\phi(x)=|x|^{2}$ it holds true that

$$
\begin{aligned}
& \frac{d}{d t} \int_{\mathbb{R}^{2}}|x|^{2} n(x, t) d x \\
= & -2 \int_{\mathbb{R}^{2}} n(x, t) x \cdot \nabla(\log n(x, t)-c(x, t)) d x \\
\leq & 2\left(\int_{\mathbb{R}^{2}}|x|^{2} n(x, t) d x\right)^{1 / 2}\left(\int_{\mathbb{R}^{2}} n(x, t)|\nabla(\log n(x, t)-c(x, t))|^{2} d x\right)^{1 / 2} .
\end{aligned}
$$

Thereby, integrating (2.19), we obtain

$$
\begin{aligned}
& \int_{\mathbb{R}^{2}}|x|^{2} n(x, t) d x \\
\leq & {\left[\left(\int_{\mathbb{R}^{2}}|x|^{2} n_{0}(x) d x\right)^{1 / 2}+\int_{0}^{t}\left(\int_{\mathbb{R}^{2}} n(x, s)|\nabla(\log n(x, s)-c(x, s))|^{2} d x\right)^{1 / 2} d s\right]^{2} } \\
\leq & 2 \int_{\mathbb{R}^{2}}|x|^{2} n_{0}(x) d x+2 t \int_{0}^{t} \int_{\mathbb{R}^{2}} n(x, s)|\nabla(\log n(x, s)-c(x, s))|^{2} d x d s,
\end{aligned}
$$

and the lemma is proved.

The proof of the previous lemma is based uniquely on the equation in $n$ and on the specific expression of the weight function defining the moment. The evolution followed by $c$ doesn't play any role, and the lemma holds true also for the (PKS) system with non-negative coefficients $\varepsilon, \eta$ and $\alpha$. Of course, one can estimate the evolution of other moments than those considered in the lemma. Here we have considered that which is most useful and used. In particular, the local-in-time bound of the weighted $L^{1}\left(\mathbb{R}^{2}, \log \left(1+|x|^{2}\right) d x\right)$ norm of $n$, together with the following lemma, will be of primary importance in obtaining the key a priori bounds and the key equiintegrability of $n$, giving the global existence with a regularizing effect.

LEMMA 2.4. Let $\psi$ be any function such that $e^{\psi} \in L^{1}\left(\mathbb{R}^{2}\right)$, and let $f$ be a non-negative function such that $\left(f \mathbf{1}_{\{f \leq 1\}}\right) \in L^{1}\left(\mathbb{R}^{2}\right) \cap L^{1}\left(\mathbb{R}^{2},|\psi(x)| d x\right)$. Then there exists a constant $C$ such that

$$
\int_{\mathbb{R}^{2}} f(x)(\log f(x))_{-} d x \leq C-\int_{\{f \leq 1\}} f(x) \psi(x) d x .
$$


Proof. Let us denote $v=f \quad \mathbf{1}_{\{f \leq 1\}}, \quad m=\int_{\mathbb{R}^{2}} v(x) \quad d x<\infty \quad$ and $\bar{v}(x)=$ $m e^{\psi(x)}\left(\int_{\mathbb{R}^{2}} e^{\psi(x)} d x\right)^{-1}$. Then, by the entropy minimization Lemma 2.1 we have

$$
\begin{aligned}
\int_{\mathbb{R}^{2}}(v(x) \log v(x)-v(x) \psi(x)) d x & \geq \int_{\mathbb{R}^{2}}(\bar{v}(x) \log \bar{v}(x)-\bar{v}(x) \psi(x)) d x \\
& =m \log m-m \log \left(\int_{\mathbb{R}^{2}} e^{\psi(x)} d x\right)=C,
\end{aligned}
$$

which gives (2.20) thanks to the identity $\int_{\mathbb{R}^{2}} f(x)(\log f(x))_{-} d x=-\int_{\mathbb{R}^{2}} v(x) \log v(x) d x$.

3. A priori estimates from the Moser-Trudinger-Onofri inequality

In [25] Onofri obtained the following sharp inequality on the sphere $\mathbb{S}^{2}$,

$$
\int_{\mathbb{S}^{2}} e^{v(s)} d s \leq \exp \left\{\int_{\mathbb{S}^{2}}\left(v(s)+\frac{1}{4}\left|\nabla_{0} v(s)\right|^{2}\right) d s\right\},
$$

for all functions $v \in L^{1}\left(\mathbb{S}^{2}, d s\right)$ such that $\left|\nabla_{0} v\right| \in L^{2}\left(\mathbb{S}^{2}, d s\right)$. Here, $d s$ is the uniform normalized surface measure on $\mathbb{S}^{2}$ so that $\int_{\mathbb{S}^{2}} d s=1$. Moreover $\nabla_{0}$ is the covariant gradient with respect to the metric $d s_{0}^{2}=d \theta^{2}+\sin ^{2} \theta d \phi^{2},(\theta, \phi)$ being the polar coordinates, i.e.

$$
\left|\nabla_{0} v\right|^{2}=\left(\frac{\partial v}{\partial \theta}\right)^{2}+(\sin \theta)^{-2}\left(\frac{\partial v}{\partial \phi}\right)^{2}
$$

With the help of the stereographic projection $\mathcal{S}$, the same inequality can be stated equivalently on $\mathbb{R}^{2}$ as follows

LEMma 3.1 (Onofri's inequality in $\mathbb{R}^{2}$ ). Let $H$ be defined as in (2.10). Then

$$
\int_{\mathbb{R}^{2}} e^{u(x)} H(x) d x \leq \exp \left\{\int_{\mathbb{R}^{2}} u(x) H(x) d x+\frac{1}{16 \pi} \int_{\mathbb{R}^{2}}|\nabla u(x)|^{2} d x\right\},
$$

for all functions $u \in L^{1}\left(\mathbb{R}^{2}, H(x) d x\right)$ such that $|\nabla u(x)| \in L^{2}\left(\mathbb{R}^{2}, d x\right)$.

Proof. It is sufficient to apply the Onofri's inequality (3.1) to the function $e^{\tilde{u}}$ with $\tilde{u}=u \circ \mathcal{S}^{-1}$; we get

$$
\begin{aligned}
\int_{\mathbb{R}^{2}} e^{u(x)} H(x) d x=\int_{\mathbb{S}^{2}} e^{\tilde{u}(s)} d s & \leq \exp \left\{\int_{\mathbb{S}^{2}}\left(\tilde{u}(s)+\frac{1}{4}\left|\nabla_{0} \tilde{u}(s)\right|^{2}\right) d s\right\} \\
& =\exp \left\{\int_{\mathbb{R}^{2}} u(x) H(x) d x+\frac{1}{16 \pi} \int_{\mathbb{R}^{2}}|\nabla u(x)|^{2} d x\right\}
\end{aligned}
$$

since $\left|\nabla_{0} \tilde{u}(s)\right|^{2}=(4 \pi H(x))^{-1}|\nabla u(x)|^{2}$ and $d s=H(x) d x$.

Thanks to inequality (3.2), we are now able to follow the first strategy giving $a$ priori estimates, namely the minimization of $\mathcal{E}(t)$ with respect to $n$. More precisely, first we apply the entropy minimization Lemma 2.1 with $\psi=(1+\delta) c+\log H$ and then we make use of the Onofri's inequality $(3.2)$ with $u=(1+\delta) c$. Working in this way, we are able to obtain the optimal threshold value, namely $8 \pi$, for the mass $M$. Observe that this is the same threshold as the one obtained in [6] for the parabolic-elliptic (PKS) system with $\varepsilon=\alpha=0$ over $\mathbb{R}^{2}$, as would be expected. Moreover, we optimize 
the result given in [23], where the author obtained the global existence of non-negative solutions $(n, c)$ of $(1.1)$ over $\mathbb{R}^{2}$ under the smallness condition $M<4 \pi$. This is due to the fact that in [23] the author used Brezis-Merle type inequality for the heat equation on $\mathbb{R}^{2}$ instead of a Moser-Trudinger type inequality as (3.2).

We give the announced estimates first formally in the following theorem. The procedure leading to the rigorous existence result will be given later in Section 6 .

Theorem 3.2 (A priori estimates). Assume $\varepsilon>0$ and $\alpha \geq 0$. Under the same hypotheses (H1)-(H2)-(H3) as in Theorem 1.1 and for sub-critical mass $M<8 \pi$, let $(n, c)$ be a non-negative solution of (1.1), supposed sufficiently smooth with fast decay as $|x| \rightarrow+\infty$. Then, $n \log n \in L^{\infty}\left(0, T ; L^{1}\left(\mathbb{R}^{2}\right)\right), n \in L^{\infty}\left(0, T ; L^{1}\left(\mathbb{R}^{2}, \log \left(1+|x|^{2}\right) d x\right)\right)$, for any $T>0$, and the following a priori estimates hold true for all $t>0$ :

(i) $\int_{\mathbb{R}^{2}} n(x, t)(\log n(x, t))_{+} d x \leq C(1+t)$;

(ii) $\int_{\mathbb{R}^{2}} n(x, t) c(x, t) d x \leq C(1+t)$;

(iii) $\|c(t)\|_{H^{1}\left(\mathbb{R}^{2}\right)}^{2} \leq C(1+t) \quad$ if $\alpha>0$, and $\|\nabla c(t)\|_{L^{2}\left(\mathbb{R}^{2}\right)}^{2} \leq C(1+t) \quad$ if $\alpha=0$;

(iv) $\mathcal{E}(t) \geq-C(1+t)$;

(v) $\mathcal{E}_{H}(t) \geq-C(M, \alpha) \quad$ if $\alpha>0$, and $\quad \mathcal{E}_{H}(t) \geq-C(1+t) \quad$ if $\alpha=0$;

(vi) $\int_{0}^{t} \int_{\mathbb{R}^{2}} n(x, s)|\nabla(\log n(x, s)-c(x, s))|^{2} d x d s \leq \mathcal{E}(0)+2 \int_{\mathbb{R}^{2}} n_{0}(x) \log \left(1+|x|^{2}\right) d x+$ $C(1+t)$

(vii) $\varepsilon \int_{0}^{t} \int_{\mathbb{R}^{2}}\left|\partial_{t} c(x, s)\right|^{2} d s \leq C(1+t)$.

Proof. Let us consider first the case $\alpha>0$. Later we will require $\delta>0$ and $\tilde{\delta}>0$ to be sufficiently small, and let us define $\bar{n}=M e^{(1+\delta) c(x, t)} H(x)\left(\int_{\mathbb{R}^{2}} e^{(1+\delta) c(x, t)} H(x) d x\right)^{-1} \cdot \quad$ We observe that, thanks to the Onofri's inequality $(3.2), c(t) \in H^{1}\left(\mathbb{R}^{2}\right)$ is sufficient in order to have $\bar{n}$ well defined and $\bar{n}(t) \in L^{1}\left(\mathbb{R}^{2}\right)$. Then, we can apply the Entropy Lemma 2.1 with $\psi=(1+\delta) c+\log H$ to obtain

$$
\begin{aligned}
E(n ;(1+\delta) c+\log H) & \geq E(\bar{n} ;(1+\delta) c+\log H) \\
& =M \log M-M \log \left(\int_{\mathbb{R}^{2}} e^{(1+\delta) c(x, t)} H(x) d x\right) .
\end{aligned}
$$

Furthermore, applying Lemma 3.1 with $u=(1+\delta) c$ to the last term in the right hand 
side of (3.3), we have for the modified free energy functional $\mathcal{E}_{H}(t)(2.10)$,

$$
\begin{array}{r}
\mathcal{E}_{H}(t)=E(n ;(1+\delta) c+\log H)+\delta \int_{\mathbb{R}^{2}} n(x, t) c(x, t) d x \\
+\frac{1}{2} \int_{\mathbb{R}^{2}}|\nabla c(x, t)|^{2} d x+\frac{\alpha}{2} \int_{\mathbb{R}^{2}} c^{2}(x, t) d x \\
\geq M \log M+\frac{1}{2}\left(1-M \frac{(1+\delta)^{2}}{8 \pi}\right) \int_{\mathbb{R}^{2}}|\nabla c(x, t)|^{2} d x-M(1+\delta) \int_{\mathbb{R}^{2}} c(x, t) H(x) d x \\
+\delta \int_{\mathbb{R}^{2}} n(x, t) c(x, t) d x+\frac{\alpha}{2} \int_{\mathbb{R}^{2}} c^{2}(x, t) d(x 3.4) \\
\geq \frac{1}{2}\left(1-M \frac{(1+\delta)^{2}}{8 \pi}\right) \int_{\mathbb{R}^{2}}|\nabla c(x, t)|^{2} d x \\
+\left(\frac{\alpha}{2}-M(1+\delta) \frac{\tilde{\delta}}{2}\right) \int_{\mathbb{R}^{2}} c^{2}(x, t) d x+M \log M \\
+\delta \int_{\mathbb{R}^{2}} n(x, t) c(x, t) d x-M(1+\delta) \frac{1}{2 \tilde{\delta}} \int_{\mathbb{R}^{2}} H^{2}(x) d x(3.5)
\end{array}
$$

Next, we choose $\delta>0$ small enough such that $M<\frac{8 \pi}{(1+\delta)^{2}}$ and $\tilde{\delta}>0$ such that $\alpha>$ $M(1+\delta) \tilde{\delta}$. This is possible because $M$ is less than the critical mass value $8 \pi$. As a consequence, since $\mathcal{E}_{H}(t)$ grows at most linearly, (3.5) gives us (ii), (iii) and (v).

When $\alpha=0$, we have to estimate differently the term $\int_{\mathbb{R}^{2}} c(x, t) H(x) d x$ in (3.4). That can be done using the following identity,

$$
\int_{\mathbb{R}^{2}} c(x, t) d x=\frac{1}{\varepsilon} M t+\int_{\mathbb{R}^{2}} c_{0}(x) d x,
$$

and the fact that $H$ is bounded. Again, whenever $|\nabla c(\cdot, t)| \in L^{2}\left(\mathbb{R}^{2}\right)$, we have that $\bar{n}$ is well defined, $\bar{n}(\cdot, t) \in L^{1}\left(\mathbb{R}^{2}\right)$, and we can obtain (ii), (iii) and (v) as before.

From now on, let $\alpha \geq 0$. Proposition 2.1, the Moment Lemma 2.3 and the definition (2.10) of $H$ give us the following estimate:

$$
\begin{aligned}
\int_{0}^{t} \int_{\mathbb{R}^{2}} n(x, s)|\nabla(\log n(x, s)-c(x, s))|^{2} d x d s \\
\leq \\
\leq \mathcal{E}(0)-\mathcal{E}(t)=\mathcal{E}(0)-\mathcal{E}_{H}(t)-\int_{\mathbb{R}^{2}} n(x, t) \log H(x) d x \\
=\mathcal{E}(0)-\mathcal{E}_{H}(t)+M \log \pi+2 \int_{\mathbb{R}^{2}} n(x, t) \log \left(1+|x|^{2}\right) d x \\
\leq \mathcal{E}(0)-\mathcal{E}_{H}(t)+M \log \pi+2 \int_{\mathbb{R}^{2}} n_{0}(x) \log \left(1+|x|^{2}\right) d x+\frac{1}{\delta} M t \\
\quad+\delta \int_{0}^{t} \int_{\mathbb{R}^{2}} n(x, s)|\nabla(\log n(x, s)-c(x, s))|^{2} d x d s .
\end{aligned}
$$

Choosing $\delta<1$ and using the lower bound (v) on $\mathcal{E}_{H}(t)$, we obtain (vi). As a consequence of (vi) and of the Moment Lemma 2.3 again, we have that $n \in$ $L^{\infty}\left(0, T ; L^{1}\left(\mathbb{R}^{2}, \log \left(1+|x|^{2}\right) d x\right)\right)$.

In the same way, we obtain (vii) and the lower bound (iv) for the free energy $\mathcal{E}(t)$, since $\varepsilon \int_{0}^{t} \int_{\mathbb{R}^{2}}\left|\partial_{t} c(s)\right|^{2} d s \leq \mathcal{E}(0)-\mathcal{E}(t)$ and $\mathcal{E}(t)=\mathcal{E}_{H}(t)+\int_{\mathbb{R}^{2}} n(x, t) \log H(x) d x$. 
To conclude, it remains to prove (i) and that $n \log n \in L^{\infty}\left(0, T ; L^{1}\left(\mathbb{R}^{2}\right)\right)$. This is a straightforward consequence of Lemma 2.4. Indeed, from the free energy definition (2.1) and the previous estimates it follows that

$$
-C(1+t) \leq \int_{\mathbb{R}^{2}} n(x, t) \log n(x, t) d x \leq C(1+t) .
$$

Next, applying Lemma 2.4 to $n$ with $\psi(x)=-(1+\delta) \log \left(1+|x|^{2}\right)$ and arbitrary $\delta>0$ in order to have $e^{\psi} \in L^{1}\left(\mathbb{R}^{2}\right)$, we have

$$
\begin{aligned}
\int_{\mathbb{R}^{2}} n(x, t)(\log n(x, t))_{+} d x & =\int_{\mathbb{R}^{2}} n(x, t) \log n(x, t) d x+\int_{\mathbb{R}^{2}} n(x, t)(\log n(x, t))_{-} d x \\
& \leq C(1+t)+(1+\delta) \int_{\mathbb{R}^{2}} n(x, t) \log \left(1+|x|^{2}\right) d x+C \leq C(1+t),
\end{aligned}
$$

and we have obtained (i). Finally, the identity

$$
\int_{\mathbb{R}^{2}}|n \log n| d x=\int_{\mathbb{R}^{2}} n \log n d x+2 \int_{\mathbb{R}^{2}} n(x, t)(\log n(x, t))_{-} d x
$$

gives us that $n \log n \in L^{\infty}\left(0, T ; L^{1}\left(\mathbb{R}^{2}\right)\right)$.

REMARK 3.3 (The critical case $M=8 \pi$ ). We cannot afford the critical mass value $M=8 \pi$ in the previous Theorem. Indeed this necessarily leads to $\delta=0$ in (3.5). Then one can prove successively estimates $(\mathrm{v}),\|c(t)\|_{L^{2}\left(\mathbb{R}^{2}\right)}^{2} \leq C(1+t)$ if $\alpha>0$, (vi), $n \in L^{\infty}\left(0, T ; L^{1}\left(\mathbb{R}^{2}, \log \left(1+|x|^{2}\right) d x\right)\right)$, (iv), (vii), but we can't obtain the fundamental estimates (i), (ii) and (iii).

Remark 3.4 (The case $\alpha=0$ ). When $\alpha=0$, in Theorem 3.2 we can use the necessary and sufficient hypothesis on $c_{0}$ to apply the Onofri's inequality (3.2), i.e. $c_{0} \in$ $L^{1}\left(\mathbb{R}^{2}, H(x) d x\right)$ instead of $c_{0} \in L^{1}\left(\mathbb{R}^{2}\right)$ and $\left|\nabla c_{0}\right| \in L^{2}\left(\mathbb{R}^{2}\right)$. However, we can obtain only estimates exponentially increasing in time. Indeed, using the identity

$$
\varepsilon \frac{d}{d t} \int_{\mathbb{R}^{2}} c(x, t) H(x) d x=\int_{\mathbb{R}^{2}} c(x, t) \Delta H(x) d x+\int_{\mathbb{R}^{2}} n(x, t) H(x) d x
$$

and the computation $\Delta H(x)=\frac{8}{\pi}\left[\frac{2|x|^{2}-1}{\left(1+|x|^{2}\right)^{4}}\right]$, we have

$$
\varepsilon \frac{d}{d t} \int_{\mathbb{R}^{2}} c(x, t) H(x) d x \leq 4 \int_{\mathbb{R}^{2}} c(x, t) H(x) d x+\frac{M}{\pi} .
$$

Then,

$$
\int_{\mathbb{R}^{2}} c(x, t) H(x) d x \leq e^{\frac{4}{\varepsilon} t}\left(\int_{\mathbb{R}^{2}} c_{0}(x) H(x) d x+\frac{M}{4 \pi}\right) .
$$

Plugging (3.7) into (3.4) instead of (3.6), we obtain the assertion.

On the other hand, under the stronger hypothesis $c_{0} \in H^{1}\left(\mathbb{R}^{2}\right)$, Theorem 3.2 holds true in the case $\alpha=0$ with the additional estimate $\int_{\mathbb{R}^{2}} c^{2}(x, t) d x \leq C\left(1+t+t^{2}\right)$ that follows from

$$
\frac{\varepsilon}{2} \int_{\mathbb{R}^{2}} c^{2}(x, t) d x \leq \frac{\varepsilon}{2} \int_{\mathbb{R}^{2}} c_{0}^{2}(x) d x+\int_{0}^{t} \int_{\mathbb{R}^{2}} n(x, t) c(x, t) d x .
$$


REMARK 3.5 (The case $\alpha=0$, corrected solutions). In the peculiar case $\alpha=0$, one can develop another sort of solution based on the following chemical deviation

$$
u(x, t):=c(x, t)-\frac{M}{8 \pi} \log H(x),
$$

with $M<8 \pi$. With this change of variable, it is sufficient to assume initially $c_{0} \in L^{1}\left(\mathbb{R}^{2}, H(x) d x\right)$ and $\nabla\left(c_{0}+\frac{M}{4 \pi} \log \left(1+|x|^{2}\right)\right) \in L^{2}\left(\mathbb{R}^{2}\right)$. The second hypothesis is hardly biologically relevant, because it breaks the non-negativity assumption on $c_{0}$. However, it allows us to solve the technical difficulty arising from evaluating the integral $\int_{\mathbb{R}^{2}} c(x, t) H(x) d x$ in (3.4) and to obtain the same estimates as in Theorem 3.2 (except for (iii), where $\nabla u$ has to be read instead for $\nabla c$ ) in the following way.

The function $\log H$ satisfies the remarkable equation

$$
-\Delta \log H(x)=2 \Delta \log \left(1+|x|^{2}\right)=8 \pi H(x) .
$$

Therefore, the pair $(n, u)$ satisfies the system

$$
\left\{\begin{aligned}
\partial_{t} n & =\Delta n-\nabla \cdot\left(n \nabla\left(u+\frac{M}{8 \pi} \log H\right)\right), \\
\varepsilon \partial_{t} u & =\Delta u+n-M H
\end{aligned}\right.
$$

which admits the following free energy

$$
\begin{array}{r}
\tilde{\mathcal{E}}(t)=\int_{\mathbb{R}^{2}} n(x, t) \log n(x, t) d x-\int_{\mathbb{R}^{2}} n(x, t) u(x, t) d x+\frac{1}{2} \int_{\mathbb{R}^{2}}|\nabla u(x, t)|^{2} d x \\
-\frac{M}{8 \pi} \int_{\mathbb{R}^{2}} n(x, t) \log H(x) d x+M \int_{\mathbb{R}^{2}} u(x, t) H(x) d x .
\end{array}
$$

It is worth noticing that the free energy $\tilde{\mathcal{E}}(t)$, up to an additional constant, is exactly the original free energy $\mathcal{E}(t)$ corresponding to the variables $(n, c)$ and that

$$
\frac{d}{d t} \tilde{\mathcal{E}}(t)=-\int_{\mathbb{R}^{2}} n(x, t)|\nabla(\log n(x, t)-c(x, t))|^{2} d x-\varepsilon \int_{\mathbb{R}^{2}}\left|\partial_{t} c(x, t)\right|^{2} d x .
$$

Then, reasoning as before, we write

$$
\begin{aligned}
& \tilde{\mathcal{E}}(t)=\delta \int_{\mathbb{R}^{2}} n(x, t) \log n(x, t) d x+(1-\delta) E\left(n ; \frac{u}{(1-\delta)}+\log H\right) \\
& +\left(1-\delta-\frac{M}{8 \pi}\right) \int_{\mathbb{R}^{2}} n(x, t) \log H(x) d x+\frac{1}{2} \int_{\mathbb{R}^{2}}|\nabla u(x, t)|^{2} d x+M \int_{\mathbb{R}^{2}} u(x, t) H(x) d x
\end{aligned}
$$

with some degree of freedom $0<\delta<1$ to be chosen later. We next apply the Entropy Lemma 2.1 and the Onofri's inequality (3.2) to obtain

$$
\begin{aligned}
& E\left(n ; \frac{u}{(1-\delta)}+\log H\right) \\
\geq & M \log M-M \log \left(\int_{\mathbb{R}^{2}} e^{u(x, t) /(1-\delta)} H(x) d x\right) \\
\geq & M \log M-\frac{M}{(1-\delta)} \int_{\mathbb{R}^{2}} u(x, t) H(x) d x-\frac{M}{16 \pi(1-\delta)^{2}} \int_{\mathbb{R}^{2}}|\nabla u(x, t)|^{2} d x .
\end{aligned}
$$


Plugging this estimate into the corrected free energy (3.12), we deduce

$$
\begin{gathered}
\tilde{\mathcal{E}}(t) \geq \delta \int_{\mathbb{R}^{2}} n(x, t) \log n(x, t) d x+\left(1-\delta-\frac{M}{8 \pi}\right) \int_{\mathbb{R}^{2}} n(x, t) \log H(x) d x \\
+\frac{1}{2}\left(1-\frac{M}{8 \pi(1-\delta)}\right) \int_{\mathbb{R}^{2}}|\nabla u(x, t)|^{2} d x+(1-\delta) M \log M .
\end{gathered}
$$

Choosing $0<\delta<1$ small enough to ensure $\left(1-\frac{M}{8 \pi(1-\delta)}\right)>0$, the bootstrap argument goes as previously. From the definition of $H$, the Moment Lemma 2.3 and the evolution Equation $(3.11)$ of $\tilde{\mathcal{E}}(t)$, we obtain

$$
\begin{aligned}
& \delta \int_{\mathbb{R}^{2}} n(x, t) \log n(x, t) d x+\frac{1}{2}\left(1-\frac{M}{8 \pi(1-\delta)}\right) \int_{\mathbb{R}^{2}}|\nabla u(x, t)|^{2} d x \\
&+\left(\frac{M}{8 \pi}+\delta\right) \int_{0}^{t} \int_{\mathbb{R}^{2}} n(x, s)|\nabla(\log n(x, s)-c(x, s))|^{2} d x d s \\
& \leq \tilde{\mathcal{E}}(0)+2\left(1-\delta-\frac{M}{8 \pi}\right) \int_{\mathbb{R}^{2}} n_{0}(x) \log \left(1+|x|^{2}\right) d x+\left(1-\delta-\frac{M}{8 \pi}\right) M t .
\end{aligned}
$$

Finally, it remains to apply Lemma 2.4 as done in Theorem 3.2 to control the contribution $\int_{\mathbb{R}^{2}} n(\log n)_{-} d x$.

\section{A priori estimates from the logarithmic HLS inequality}

In this section we follow the strategy dual to the previous one, starting from the minimization of the modified free energy functional $\mathcal{E}_{H}(t)$ with respect to $c$ and giving the same a priori estimates. The role of the Onofri's inequality (3.2) will be played by the logarithmic HLS inequality below when $\alpha=0$ and by its generalization to the Bessel kernel $B_{\alpha}$ when $\alpha>0$ (see Lemma 4.2). Therefore, the cases $\alpha>0$ and $\alpha=0$ have to be treated separately again.

Lemma 4.1 (Logarithmic Hardy-Littlewood-Sobolev inequality in $\mathbb{R}^{2}$ ). For all non-negative functions $f \in L^{1}\left(\mathbb{R}^{2}\right)$ such that $\int_{\mathbb{R}^{2}} f(x) d x=M, \int_{\mathbb{R}^{2}} f(x) \log (1+$ $\left.|x|^{2}\right) d x<\infty$ and $\int_{\mathbb{R}^{2}} f(x) \log f(x) d x$ is finite, the following inequality holds true:

$$
-\int_{\mathbb{R}^{2}} \int_{\mathbb{R}^{2}} f(x) \log |x-y| f(y) d x d y \leq \frac{M}{2} \int_{\mathbb{R}^{2}} f(x) \log f(x) d x+C(M) .
$$

The proof of Lemma 4.1 can be found for example in [8]. Let us observe here that the logarithmic HLS inequality (4.1) written equivalently on $\mathbb{S}^{2}$ is dual to the Onofri's inequality (3.1) in the sense that extremal functions for one inequality determine extremal functions for the other inequality (see [4], [8]). As an example of this kind of duality result, we show in Appendix A.4 how one can directly obtain inequality (4.2) from the Onofri's inequality on $\mathbb{R}^{2}(3.2)$.

The generalization of inequality (4.1) to the Bessel kernel $B_{\alpha}$ (whose definition is given in Lemma 2.2) is the following Lemma 4.2. One possible interpretation why an additional "logarithmic" momentum appears in (4.2) is that the homogeneity of the logarithmic kernel $-\log |z|$ has been broken in the Bessel potential $B_{\alpha}$ (consider for instance the dilation $f_{\lambda}(x)=\lambda f(\lambda x)$ ). It shares indeed the same singularity for small $|z|$, but for large $|z|, B_{\alpha}$ is a positive exponentially decreasing function. For other extensions of Lemma 4.1, see [4]. 
Lemma 4.2. For all non-negative functions $f \in L^{1}\left(\mathbb{R}^{2}\right)$ such that $\int_{\mathbb{R}^{2}} f(x) d x=M$, $\int_{\mathbb{R}^{2}} f(x) \log f(x) d x$ is finite and $\int_{\mathbb{R}^{2}} f(x) \log \left(1+|x|^{2}\right) d x<\infty$, the following inequality holds true:

$$
\begin{aligned}
\int_{\mathbb{R}^{2}} \int_{\mathbb{R}^{2}} f(x) B_{\alpha}(x-y) f(y) d x d y \leq & \frac{M}{4 \pi} \int_{\mathbb{R}^{2}} f(x) \log f(x) d x \\
& +\frac{M}{2 \pi} \int_{\mathbb{R}^{2}} f(x) \log \left(1+|x|^{2}\right) d x+C(M) .
\end{aligned}
$$

Proof. The Bessel kernel $B_{\alpha}$ is a positive radial decreasing function such that $B_{\alpha}(z) \rightarrow+\infty$ when $|z| \rightarrow 0$ as $\frac{1}{2 \pi} \log \left(\frac{1}{|z|}\right)+O(1)$. We only prove below the upper side of this asymptotic estimate, which is sufficient for our purpose. Indeed, following [14], $B_{\alpha}$ can be written as the sum of the three integrals $\int_{0}^{r^{2}}+\int_{r^{2}}^{1}+\int_{1}^{+\infty}$, with $r=|z|<1$. For the first and third integrals we have, respectively,

$$
\frac{1}{4 \pi} \int_{0}^{r^{2}} \frac{1}{t} e^{-\frac{r^{2}}{4 t}-\alpha t} d t \leq \frac{1}{4 \pi} \int_{0}^{r^{2}} \frac{1}{t} e^{-\frac{r^{2}}{4 t}} d t=\frac{1}{4 \pi} \int_{\frac{1}{4}}^{+\infty} \frac{e^{-y}}{y} d y<\infty
$$

and

$$
\frac{1}{4 \pi} \int_{1}^{+\infty} \frac{1}{t} e^{-\frac{r^{2}}{4 t}-\alpha t} d t \leq \frac{1}{4 \pi} \int_{1}^{+\infty} e^{-\alpha t} d t<\infty .
$$

The second integral satisfies

$$
\frac{1}{4 \pi} e^{-\left(\alpha+\frac{1}{4}\right)} \log \left(\frac{1}{r^{2}}\right) \leq \frac{1}{4 \pi} \int_{r^{2}}^{1} \frac{1}{t} e^{-\frac{r^{2}}{4 t}-\alpha t} d t \leq \frac{1}{4 \pi} e^{-r^{2}\left(\alpha+\frac{1}{4}\right)} \log \left(\frac{1}{r^{2}}\right) .
$$

As a consequence of $(4.3), \lim _{|z| \rightarrow 0} B_{\alpha}(z)=+\infty$, and

$$
\begin{aligned}
\int_{\mathbb{R}^{2}} \int_{\mathbb{R}^{2}} f(x) B_{\alpha}(x-y) f(y) d x d y \leq & \int_{\mathbb{R}^{2}} \int_{|x-y| \leq 1} f(x)\left(C-\frac{1}{2 \pi} \log |x-y|\right) f(y) d x d y \\
& +\int_{\mathbb{R}^{2}} \int_{|x-y|>1} f(x) B_{\alpha}(x-y) f(y) d x d y \\
\leq C M^{2}- & \frac{1}{2 \pi} \int_{\mathbb{R}^{2}} \int_{\mathbb{R}^{2}} f(x) \log |x-y| f(y) d x d y \\
& +\frac{1}{2 \pi} \int_{\mathbb{R}^{2}} \int_{|x-y|>1} f(x) \log |x-y| f(y) d x d y .
\end{aligned}
$$

Using the inequality

$$
\log |x-y| \leq \frac{1}{2} \log 2+\frac{1}{2} \log \left(1+|x|^{2}\right)+\frac{1}{2} \log \left(1+|y|^{2}\right),
$$

it follows that

$$
\frac{1}{2 \pi} \int_{\mathbb{R}^{2}} \int_{|x-y|>1} f(x) \log |x-y| f(y) d x d y \leq C M^{2}+\frac{M}{2 \pi} \int_{\mathbb{R}^{2}} f(x) \log \left(1+|x|^{2}\right) d x .
$$

Applying the logarithmic HLS inequality (4.1), we obtain (4.2). 
Proof. [Proof of Theorem 3.2.] We first consider the case $\alpha>0$. Let $0<\delta<1$, to be chosen later, and let $\bar{c}$ be the quasi-stationary state $(2.12)$ corresponding to $(1-\delta)^{-1} n$, i.e., $\bar{c}(x, t)=\frac{1}{(1-\delta)}\left(B_{\alpha} * n(t)\right)(x)$. Then, by the minimization Lemma 2.2, the chemical energy (2.3) satisfies

$$
F_{\alpha}\left(c ; \frac{n}{1-\delta}\right) \geq F_{\alpha}\left(\bar{c} ; \frac{n}{1-\delta}\right)=-\frac{1}{2(1-\delta)} \int_{\mathbb{R}^{2}} n(x, t) \bar{c}(x, t) d x, \quad \alpha>0
$$

This is possible as soon as $n(t) \in L^{1}\left(\mathbb{R}^{2}\right)$ and $n(t) \log n(t) \in L^{1}\left(\mathbb{R}^{2}\right)$. Next, from (4.5) and Lemma 4.2 we obtain

$$
\begin{aligned}
\mathcal{E}(t)=\int_{\mathbb{R}^{2}} n(x, t) \log n(x, t) d x & +(1-\delta) F_{\alpha}\left(c ; \frac{n}{1-\delta}\right) \\
& +\delta\left(\frac{1}{2} \int_{\mathbb{R}^{2}}|\nabla c(x, t)|^{2} d x+\frac{\alpha}{2} \int_{\mathbb{R}^{2}} c^{2}(x, t) d x\right) \\
\geq \int_{\mathbb{R}^{2}} n(x, t) \log n(x, t) d x & -\frac{1}{2(1-\delta)} \iint_{\mathbb{R}^{2} \times \mathbb{R}^{2}} n(x, t) B_{\alpha}(x-y) n(y, t) d x d y \\
& +\delta\left(\frac{1}{2} \int_{\mathbb{R}^{2}}|\nabla c(x, t)|^{2} d x+\frac{\alpha}{2} \int_{\mathbb{R}^{2}} c^{2}(x, t) d x\right) \\
\geq\left(1-\frac{M}{8 \pi(1-\delta)}\right) \int_{\mathbb{R}^{2}} n(x, t) \log n(x, t) d x-\frac{1}{4 \pi(1-\delta)} \int_{\mathbb{R}^{2}} n(x, t) \log \left(1+|x|^{2}\right) d x & -\frac{1}{1-\delta} C(M)+\delta\left(\frac{1}{2} \int_{\mathbb{R}^{2}}|\nabla c(x, t)|^{2} d x+\frac{\alpha}{2} \int_{\mathbb{R}^{2}} c^{2}(x, t) d x\right) .
\end{aligned}
$$

Moreover, using the definition (2.10) of $H$ it follows that

$$
\begin{array}{rl}
\mathcal{E}(t) \geq\left(1-\frac{M}{8 \pi(1-\delta)}\right) \int_{\mathbb{R}^{2}} & n(x, t) \log n(x, t) d x+\frac{M}{8 \pi(1-\delta)} \int_{\mathbb{R}^{2}} n(x, t) \log H(x) d x \\
& +\delta\left(\frac{1}{2} \int_{\mathbb{R}^{2}}|\nabla c(x, t)|^{2} d x+\frac{\alpha}{2} \int_{\mathbb{R}^{2}} c^{2}(x, t) d x\right)-\frac{1}{1-\delta} C(M) .
\end{array}
$$

Therefore, the modified free energy $\mathcal{E}_{H}(t)$ verifies

$$
\begin{aligned}
\mathcal{E}_{H}(t) \geq\left(1-\frac{M}{8 \pi(1-\delta)}\right) & \int_{\mathbb{R}^{2}} n(x, t) \log \left(\frac{n(x, t)}{H(x)}\right) d x \\
& +\delta\left(\frac{1}{2} \int_{\mathbb{R}^{2}}|\nabla c(x, t)|^{2} d x+\frac{\alpha}{2} \int_{\mathbb{R}^{2}} c^{2}(x, t) d x\right)-\frac{1}{1-\delta} C(M),
\end{aligned}
$$

and the theorem follows from the above estimate. Indeed, since

$$
\int_{\mathbb{R}^{2}} n(x, t) \log \left(\frac{n(x, t)}{H(x)}\right) d x=\int_{\mathbb{R}^{2}}\left(\frac{n(x, t)}{H(x)}\right) \log \left(\frac{n(x, t)}{H(x)}\right) H(x) d x \geq-e^{-1}
$$

choosing $0<\delta<1$ such that $M<8 \pi(1-\delta)$ and using the facts that $\mathcal{E}_{H}(t)$ grows at most linearly, estimates (iii) and (v) with $\alpha>0$ of Theorem 3.2 follow. The remaining estimates of Theorem 3.2 follow as in Section 3.

To conclude, let us consider the case $\alpha=0$. In this case, the minimization principle (4.5) does not hold true, since $\nabla \bar{c}(t)=\nabla E_{2} * n(t)$ does not lie in $L^{2}\left(\mathbb{R}^{2}\right)$ in general. Nevertheless we can obtain the required estimates by considering the corrected quasistationary state $\bar{c}(x, t)=\frac{1}{(1-\delta)}\left(E_{2} *(n(t)-M H)\right)(x)$, whose gradient does belong to 
$L^{2}\left(\mathbb{R}^{2}\right)$ by Lemma 2.2. Indeed, choosing $f_{1}=n$ and $f_{2}=-M H$, the hypotheses of Lemma 2.2 are satisfied as soon as $n(t) \in L^{1}\left(\mathbb{R}^{2}\right), n(t) \log n(t) \in L^{1}\left(\mathbb{R}^{2}\right)$ and $n(t) \in$ $L^{1}\left(\mathbb{R}^{2}, \log \left(1+|x|^{2}\right) d x\right)$. Hence,

$$
\begin{aligned}
F_{0}\left(c ; \frac{1}{1-\delta}(n-M H)\right) & \geq F_{0}\left(\bar{c} ; \frac{1}{1-\delta}(n-M H)\right) \\
& =-\frac{1}{2(1-\delta)} \int_{\mathbb{R}^{2}}(n(x, t)-M H(x)) \bar{c}(x, t) d x .
\end{aligned}
$$

Then, acting as before, we obtain

$$
\begin{aligned}
\mathcal{E}(t)= & \int_{\mathbb{R}^{2}} n(x, t) \log n(x, t) d x+(1-\delta) F_{0}\left(c ;(1-\delta)^{-1}(n-M H)\right) \\
& +\frac{\delta}{2} \int_{\mathbb{R}^{2}}|\nabla c(x, t)|^{2} d x-M \int_{\mathbb{R}^{2}} c(x, t) H(x) d x \\
\geq & \int_{\mathbb{R}^{2}} n(x, t) \log n(x, t) d x \\
& +\frac{1}{4 \pi(1-\delta)} \iint_{\mathbb{R}^{2} \times \mathbb{R}^{2}}(n(x, t)-M H(x)) \log |x-y|(n(y, t)-M H(y)) d x d y \\
& +\frac{\delta}{2} \int_{\mathbb{R}^{2}}|\nabla c(x, t)|^{2} d x-M \int_{\mathbb{R}^{2}} c(x, t) H(x) d x \\
= & \int_{\mathbb{R}^{2}} n(x, t) \log n(x, t) d x+\frac{1}{4 \pi(1-\delta)} \iint_{\mathbb{R}^{2} \times \mathbb{R}^{2}} n(x, t) \log |x-y| n(y, t) d x d y \\
- & \frac{M}{2 \pi(1-\delta)} \iint_{\mathbb{R}^{2} \times \mathbb{R}^{2}} H(x) \log |x-y| n(y, t) d x d y \\
+ & \frac{M^{2}}{4 \pi(1-\delta)} \iint_{\mathbb{R}^{2} \times \mathbb{R}^{2}} H(x) \log |x-y| H(y) d x d y \\
+ & \frac{\delta}{2} \int_{\mathbb{R}^{2}}|\nabla c(x, t)|^{2} d x-M \int_{\mathbb{R}^{2}} c(x, t) H(x) d x .
\end{aligned}
$$

Using inequality (4.4), it is straightforward to prove that

$$
\begin{gathered}
-\frac{M}{2 \pi(1-\delta)} \iint_{\mathbb{R}^{2} \times \mathbb{R}^{2}} H(x) \log |x-y| n(y, t) d x d y \\
\geq-\frac{M}{4 \pi(1-\delta)} \int_{\mathbb{R}^{2}} n(x, t) \log \left(1+|x|^{2}\right) d x-\frac{1}{1-\delta} C(M) .
\end{gathered}
$$

Finally, applying the logarithmic HLS inequality (4.1) to $n$, we arrive exactly at the estimate

$$
\begin{gathered}
\mathcal{E}(t) \geq\left(1-\frac{M}{8 \pi(1-\delta)}\right) \int_{\mathbb{R}^{2}} n(x, t) \log n(x, t) d x+\frac{M}{8 \pi(1-\delta)} \int_{\mathbb{R}^{2}} n(x, t) \log H(x) d x \\
\quad+\frac{1}{2} \delta \int_{\mathbb{R}^{2}}|\nabla c(x, t)|^{2} d x-M \int_{\mathbb{R}^{2}} c(x, t) H(x) d x-\frac{1}{1-\delta} C(M),
\end{gathered}
$$

and the proof follows as in Section 3 .

REMARK 4.3. Again, we see here that the mass $M$ cannot equal the critical value $8 \pi$ (see Remark 3.3). 


\section{Regularizing effect}

In the previous section it has been proved implicitly that under the hypothesis of Theorem 3.2; in particular, for sub-critical mass $M$, the solution $n$ of system (1.1) is locally in time equi-integrable, i.e., there exists a modulus of equi-integrability $\omega(T ; k)$, $T>0$ and $k>0$ such that

$$
\sup _{0 \leq t \leq T} \int_{\mathbb{R}^{2}}(n(x, t)-k)_{+} d x \leq \omega(T ; k) \quad \text { and } \quad \lim _{k \rightarrow+\infty} \omega(T ; k)=0 .
$$

Indeed, obviously $\int_{\mathbb{R}^{2}}(n(x, t)-k)_{+} d x \leq M$ for any $k>0$, while for $k>1$ we have

$$
\begin{aligned}
\int_{\mathbb{R}^{2}}(n(x, t)-k)_{+} d x & \leq \frac{1}{\log k} \int_{\mathbb{R}^{2}}(n(x, t)-k)_{+} \log n(x, t) d x \\
& \leq \frac{1}{\log k} \int_{\mathbb{R}^{2}} n(x, t)(\log n(x, t))_{+} d x \leq \frac{C(1+t)}{\log k} .
\end{aligned}
$$

In this section, following a now classical idea initiated in [19], we will obtain $a$ priori estimates for the $L^{p}$-norm of $n$, with the help of the equi-integrability property (5.1) and of the fact proved in Theorem 3.2 that $\partial_{t} c \in L^{2}\left(0, T ; L^{2}\left(\mathbb{R}^{2}\right)\right)$. Since the hypothesis $\left\|n_{0}\right\|_{L^{p}\left(\mathbb{R}^{2}\right)}<\infty$ is not required, the following result is an hypercontractivitytype result.

TheOREM 5.1. Let $T>0$ and $1<p<\infty$. Under the hypothesis of Theorem 3.2, there exists a constant $C(T)$ not depending on $\left\|n_{0}\right\|_{L^{p}\left(\mathbb{R}^{2}\right)}$ such that

$$
\int_{\mathbb{R}^{2}} n^{p}(x, t) d x \leq C(T)\left(1+t^{1-p}\right), \quad \forall 0<t \leq T,
$$

i.e., the cell density $n(\cdot, t)$ belongs to $L^{p}\left(\mathbb{R}^{2}\right)$ for any positive time $t$.

Proof. Let $k>0$, to be chosen later. We derive a non-linear differential inequality, for the quantity $Y_{p}(t):=\int_{\mathbb{R}^{2}}(n(x, t)-k)_{+}^{p} d x$, which guarantees that the $L^{p}$-norm of $n$ remains finite whatever $\left\|n_{0}\right\|_{L^{p}\left(\mathbb{R}^{2}\right)}$ is (possibly infinite).

First step: the differential inequality. Multiplying the equation in $n$ in (1.1) by $p(n-k)_{+}^{p-1}$ yields, after integration by parts,

$$
\begin{array}{r}
\frac{d}{d t} \int_{\mathbb{R}^{2}}(n-k)_{+}^{p} d x=-4 \frac{(p-1)}{p} \int_{\mathbb{R}^{2}}\left|\nabla(n-k)_{+}^{p / 2}\right|^{2} d x-(p-1) \int_{\mathbb{R}^{2}}(n-k)_{+}^{p} \Delta c d x \\
-p k \int_{\mathbb{R}^{2}}(n-k)_{+}^{p-1} \Delta c d x
\end{array}
$$

There is some subtlety hidden, here because we cannot directly use $-\Delta c=n-\alpha c$ as for the parabolic-elliptic system $(\varepsilon=0)$. However, using the equation in $c$, one obtains

$$
\begin{aligned}
& \frac{d}{d t} \int_{\mathbb{R}^{2}}(n-k)_{+}^{p} d x \\
\leq & -4 \frac{(p-1)}{p} \int_{\mathbb{R}^{2}}\left|\nabla(n-k)_{+}^{p / 2}\right|^{2} d x \\
& +(p-1) \int_{\mathbb{R}^{2}}(n-k)_{+}^{p+1} d x+(2 p-1) k \int_{\mathbb{R}^{2}}(n-k)_{+}^{p} d x+p k^{2} \int_{\mathbb{R}^{2}}(n-k)_{+}^{p-1} d x \\
& -\varepsilon(p-1) \int_{\mathbb{R}^{2}}(n-k)_{+}^{p} \partial_{t} c d x-\varepsilon p k \int_{\mathbb{R}^{2}}(n-k)_{+}^{p-1} \partial_{t} c d x,
\end{aligned}
$$


and the additional non-linear terms $\int_{\mathbb{R}^{2}}(n-k)_{+}^{p} \partial_{t} c d x$ and $\int_{\mathbb{R}^{2}}(n-k)_{+}^{p-1} \partial_{t} c d x$ can be estimated in the following way. Using the Gagliardo-Nirenberg-Sobolev inequality

$$
\int_{\mathbb{R}^{2}} u^{4}(x) d x \leq C \int_{\mathbb{R}^{2}} u^{2}(x) d x \int_{\mathbb{R}^{2}}|\nabla u(x)|^{2} d x,
$$

with $u=(n-k)_{+}^{p / 2}$, we obtain

$$
\begin{aligned}
\left|\int_{\mathbb{R}^{2}}(n-k)_{+}^{p} \partial_{t} c d x\right| & \leq\left(\int_{\mathbb{R}^{2}}(n-k)_{+}^{2 p} d x\right)^{1 / 2}\left\|\partial_{t} c\right\|_{L^{2}\left(\mathbb{R}^{2}\right)} \\
& \leq C\left(\int_{\mathbb{R}^{2}}(n-k)_{+}^{p} d x\right)^{1 / 2}\left(\int_{\mathbb{R}^{2}}\left|\nabla(n-k)_{+}^{p / 2}\right|^{2} d x\right)^{1 / 2}\left\|\partial_{t} c\right\|_{L^{2}\left(\mathbb{R}^{2}\right)} \\
& \leq \varepsilon C(p)\left\|\partial_{t} c\right\|_{L^{2}\left(\mathbb{R}^{2}\right)}^{2} \int_{\mathbb{R}^{2}}(n-k)_{+}^{p} d x+\frac{2}{\varepsilon p} \int_{\mathbb{R}^{2}}\left|\nabla(n-k)_{+}^{p / 2}\right|^{2} d x .
\end{aligned}
$$

On the other hand, by interpolation and the same Gagliardo-Nirenberg-Sobolev inequality as above, we have for $p \geq \frac{3}{2}$ that

$$
\begin{aligned}
\left|\int_{\mathbb{R}^{2}}(n-k)_{+}^{p-1} \partial_{t} c d x\right| & \leq\left(\int_{\mathbb{R}^{2}}(n-k)_{+}^{2(p-1)} d x\right)^{1 / 2}\left\|\partial_{t} c\right\|_{L^{2}\left(\mathbb{R}^{2}\right)} \\
& \leq\left(C(M, p)+C(p) \int_{\mathbb{R}^{2}}(n-k)_{+}^{2 p} d x\right)^{1 / 2}\left\|\partial_{t} c\right\|_{L^{2}\left(\mathbb{R}^{2}\right)} \\
& \leq C(M, p)\left\|\partial_{t} c\right\|_{L^{2}\left(\mathbb{R}^{2}\right)}+\varepsilon C(p, k)\left\|\partial_{t} c\right\|_{L^{2}\left(\mathbb{R}^{2}\right)}^{2} \int_{\mathbb{R}^{2}}(n-k)_{+}^{p} d x \\
& +\frac{(p-1)}{\varepsilon p^{2} k} \int_{\mathbb{R}^{2}}\left|\nabla(n-k)_{+}^{p / 2}\right|^{2} d x .
\end{aligned}
$$

Inserting (5.5) and (5.6) in (5.4) gives for $p \geq \frac{3}{2}$ that

$$
\begin{aligned}
& \frac{d}{d t} \int_{\mathbb{R}^{2}}(n-k)_{+}^{p} d x \\
\leq & -\frac{(p-1)}{p} \int_{\mathbb{R}^{2}}\left|\nabla(n-k)_{+}^{p / 2}\right|^{2} d x \\
& +(p-1) \int_{\mathbb{R}^{2}}(n-k)_{+}^{p+1} d x+(2 p-1) k \int_{\mathbb{R}^{2}}(n-k)_{+}^{p} d x+p k^{2} \int_{\mathbb{R}^{2}}(n-k)_{+}^{p-1} d x \\
& +\varepsilon C\left\|\partial_{t} c\right\|_{L^{2}\left(\mathbb{R}^{2}\right)}^{2} \int_{\mathbb{R}^{2}}(n-k)_{+}^{p} d x+C\left\|\partial_{t} c\right\|_{L^{2}\left(\mathbb{R}^{2}\right)}
\end{aligned}
$$

Next, we estimate the nonlinear and negative contribution $-\frac{(p-1)}{p} \int_{\mathbb{R}^{2}}\left|\nabla(n-k)_{+}^{p / 2}\right|^{2} d x$ in terms of $\int_{\mathbb{R}^{2}}(n-k)_{+}^{p+1} d x$ and of the modulus of equi-integrability $\omega(T ; k)$, with the help of the Sobolev's inequality. Indeed,

$$
\begin{aligned}
\int_{\mathbb{R}^{2}}(n-k)_{+}^{p+1} d x & =\int_{\mathbb{R}^{2}}\left((n-k)_{+}^{\frac{(p+1)}{2}}\right)^{2} d x \leq C\left(\int_{\mathbb{R}^{2}}\left|\nabla(n-k)_{+}^{\frac{(p+1)}{2}}\right| d x\right)^{2} \\
& =C(p)\left(\int_{\mathbb{R}^{2}}(n-k)_{+}^{\frac{1}{2}}\left|\nabla(n-k)_{+}^{\frac{p}{2}}\right| d x\right)^{2} \\
& \leq C(p) \int_{\mathbb{R}^{2}}(n-k)_{+} d x \int_{\mathbb{R}^{2}}\left|\nabla(n-k)_{+}^{p / 2}\right|^{2} d x \\
& \leq C(p) \omega(T ; k) \int_{\mathbb{R}^{2}}\left|\nabla(n-k)_{+}^{p / 2}\right|^{2} d x, \quad \forall 0<t \leq T .
\end{aligned}
$$


Moreover, since for $p \geq 2$ it holds true that

$$
\int_{\mathbb{R}^{2}}(n-k)_{+}^{p-1} d x \leq \int_{\mathbb{R}^{2}}(n-k)_{+} d x+\int_{\mathbb{R}^{2}}(n-k)_{+}^{p} d x,
$$

inserting (5.8) and (5.9) in (5.7) gives for $p \geq 2$ and $0<t \leq T$ that

$$
\begin{aligned}
\frac{d}{d t} \int_{\mathbb{R}^{2}}(n-k)_{+}^{p} d x \leq & (p-1)\left(1-\frac{1}{p C(p) \omega(T ; k)}\right) \int_{\mathbb{R}^{2}}(n-k)_{+}^{p+1} d x \\
& +C\left(1+\varepsilon\left\|\partial_{t} c\right\|_{L^{2}\left(\mathbb{R}^{2}\right)}^{2}\right) \int_{\mathbb{R}^{2}}(n-k)_{+}^{p} d x+C\left\|\partial_{t} c\right\|_{L^{2}\left(\mathbb{R}^{2}\right)}+p k^{2} M
\end{aligned}
$$

Finally, for any fixed $p$ we choose $k=k(p, T)$ sufficiently large such that

$$
\delta:=\frac{1}{p C(p) \omega(T ; k(p, T))}-1>0 .
$$

This is clearly possible because $\omega(T ; k) \rightarrow 0$ as $k \rightarrow+\infty$. For such a $k$, using the interpolation

$$
\begin{aligned}
\int_{\mathbb{R}^{2}}(n-k)_{+}^{p} d x & \leq\left(\int_{\mathbb{R}^{2}}(n-k)_{+} d x\right)^{\frac{1}{p}}\left(\int_{\mathbb{R}^{2}}(n-k)_{+}^{p+1} d x\right)^{\left(1-\frac{1}{p}\right)} \\
& \leq M^{\frac{1}{p}}\left(\int_{\mathbb{R}^{2}}(n-k)_{+}^{p+1} d x\right)^{\left(1-\frac{1}{p}\right)}
\end{aligned}
$$

we end up with the following differential inequality for $Y_{p}(t), p \geq 2$ fixed and $0<t \leq T$ :

$$
\begin{array}{r}
\frac{d}{d t} Y_{p}(t) \leq-(p-1) M^{-\frac{1}{p-1}} \delta Y_{p}^{\beta}(t)+C_{1}\left(1+\varepsilon\left\|\partial_{t} c(t)\right\|_{L^{2}\left(\mathbb{R}^{2}\right)}^{2}\right) Y_{p}(t) \\
+C_{2}\left(1+\varepsilon\left\|\partial_{t} c(t)\right\|_{L^{2}\left(\mathbb{R}^{2}\right)}^{2}\right)
\end{array}
$$

where $\beta=\frac{p}{p-1}>1$.

Second step: estimate on $Y_{p}, p \geq 2$. Let us write the differential inequality (5.12) as follows for simplicity:

$$
\frac{d}{d t} Y_{p}(t) \leq-\gamma Y_{p}^{\beta}(t)+f(t) Y_{p}(t)+f(t), \quad 0<t \leq T,
$$

where $\quad \gamma=(p-1) M^{-\frac{1}{p-1}} \delta>0 \quad$ and $\quad f(t)=\bar{C}\left(1+\varepsilon\left\|\partial_{t} c(t)\right\|_{L^{2}\left(\mathbb{R}^{2}\right)}^{2}\right) \quad$ with $\quad \bar{C}=$ $\max \left\{C_{1}, C_{2}\right\}$. Next we show that there exists a constant $C(T)$ not depending on $Y_{p}(0)$ such that

$$
Y_{p}(t) \leq C(T) \frac{1}{t^{p-1}}, \quad 0<t \leq T
$$

by comparison of $Y_{p}(t)$ with positive solutions of the differential equation

$$
\frac{d}{d t} Z_{p}(t)=-\gamma Z_{p}^{\beta}(t)+f(t) Z_{p}(t)+f(t), \quad 0<t \leq T .
$$


To do that, let $Z_{p}$ be a positive solution of $(5.15)$ with $Z_{p}(0) \geq\left(\frac{\bar{C}}{\gamma}\right)^{1 /(\beta-1)}$ (such a solution exists from Carathéodory regularity of ordinary differential equations). Because $f(t)$ is positive, $Z_{p}$ satisfies the differential inequality

$$
\frac{d}{d t} Z_{p}(t) \geq-\gamma Z_{p}^{\beta}(t)+\bar{C} Z_{p}(t), \quad 0<t \leq T
$$

Therefore, since $\left(\frac{\bar{C}}{\gamma}\right)^{1 /(\beta-1)}$ is a constant solution of $Z^{\prime}(t)=-\gamma Z^{\beta}(t)+\bar{C} Z(t)$, by comparison in (5.16) we get $Z_{p}(t) \geq\left(\frac{\bar{C}}{\gamma}\right)^{1 /(\beta-1)}, \forall 0 \leq t \leq T$. As a consequence, from (5.15) $Z_{p}$ satisfies also the differential inequality

$$
\frac{d}{d t} Z_{p}(t) \leq-\gamma Z_{p}^{\beta}(t)+h(t) Z_{p}(t), \quad 0 \leq t \leq T,
$$

where $h(t)=\left(1+\left(\gamma \bar{C}^{-1}\right)^{1 /(\beta-1)}\right) f(t)$. Integrating $(5.17)$ over $(0, t)$, it is straightforward to prove that

$$
Z_{p}^{1-\beta}(t) \geq Z_{p}^{1-\beta}(0) e^{(1-\beta) \int_{0}^{t} h(\tau) d \tau}+\gamma(\beta-1) \int_{0}^{t} e^{(1-\beta) \int_{s}^{t} h(\tau) d \tau} d s, \quad 0 \leq t \leq T .
$$

Then, thanks to the estimate (vi) in Theorem 3.2 and by the definition of $h(t)$, it holds true that

$$
\begin{aligned}
& Z_{p}^{1-\beta}(t) \geq Z_{p}^{1-\beta}(0) e^{C(1-\beta) \varepsilon \int_{0}^{T}\left\|\partial_{t} c(s)\right\|_{L^{2}\left(\mathbb{R}^{2}\right)}^{2} d s} e^{C(1-\beta) t}+\frac{\gamma}{C} e^{C(1-\beta) \varepsilon \int_{0}^{T}\left\|\partial_{t} c(s)\right\|_{L^{2}\left(\mathbb{R}^{2}\right)}^{2} d s}\left(1-e^{C(1-\beta) t}\right) \\
& \geq \gamma(\beta-1) e^{C(1-\beta)(1+T)} e^{C(1-\beta) T} t=C(T) t, \quad 0 \leq t \leq T,
\end{aligned}
$$

and therefore

$$
Z_{p}(t) \leq C(T) \frac{1}{t^{p-1}}, \quad 0 \leq t \leq T
$$

where $C(T)$ does not depend on $Z_{p}(0)$.

Finally, let $Z_{p}$ be a positive solution of (5.15) with $Z_{p}(0)=$ $\max \left\{\left(\frac{\bar{C}}{\gamma}\right)^{1 /(\beta-1)}, Y_{p}(0)\right\}$. Again by comparison, $Y_{p}(t) \leq Z_{p}(t) \forall 0<t \leq T$, and (5.14) follows by (5.18).

Third step: $L^{p}$ regularity of $n$. To conclude, it is sufficient to observe that for any $k>0$ we have

$$
\begin{aligned}
\int_{\mathbb{R}^{2}} n^{p}(x, t) d x & =\int_{\{n \leq 2 k\}} n^{p}(x, t) d x+\int_{\{n>2 k\}} n^{p}(x, t) d x \\
& \leq(2 k)^{p-1} M+2^{p} \int_{\{n>2 k\}}(n(x, t)-k)^{p} d x \\
& \leq(2 k)^{p-1} M+2^{p} \int_{\mathbb{R}^{2}}(n(x, t)-k)_{+}^{p} d x
\end{aligned}
$$

where the inequality $x^{p} \leq 2^{p}(x-k)^{p}$, for $x \geq 2 k$, has been used. Therefore, estimate (5.2) follows for any $p \geq 2$ by (5.14) and (5.19) choosing $k=k(p, T)$ sufficiently large such that (5.11) holds true. For $1<p<2$, the theorem follows by interpolation. 


\section{Global existence}

We are now able to prove Theorem 1.1 collecting all the estimates proved in the previous sections. In order to do that, we need first to regularize the chemotaxis system (1.1) and then to prove that the a priori estimates hold true and pass to the limit. Since this procedure is quite technical and usual, we just sketch the proof. For the parabolic-elliptic case with $\alpha=0$ one can consult for example [6], where the regularizing procedure has been written in full detail.

The regularized system that we consider is

$$
\left\{\begin{array}{c}
\frac{\partial n^{\sigma}}{\partial t}=\Delta n^{\sigma}-\nabla \cdot\left(n^{\sigma} \nabla c^{\sigma}\right), \quad t>0, x \in \mathbb{R}^{2}, \\
\varepsilon \frac{\partial c^{\sigma}}{\partial t}=\Delta c^{\sigma}+n^{\sigma} * \rho^{\sigma}-\alpha c^{\sigma}, \quad t>0, x \in \mathbb{R}^{2}, \\
n^{\sigma}(\cdot, 0)=n_{0} * \rho^{\sigma}, \quad c^{\sigma}(\cdot, 0)=c_{0} * \rho^{\sigma}, \quad x \in \mathbb{R}^{2},
\end{array}\right.
$$

for some regularizing kernel $\rho^{\sigma}(x)=\frac{1}{\sigma^{2}} \rho\left(\frac{x}{\sigma}\right)$ with $\rho \in \mathcal{D}^{+}\left(\mathbb{R}^{2}\right)$ and $\int_{\mathbb{R}^{2}} \rho(x) d x=1$. The first step is to prove the global existence of a smooth solution $\left(n^{\sigma}, c^{\sigma}\right)$ of $(6.1)$. This result can be obtained through a Picard fixed-point method, writing $\left(n^{\sigma}, c^{\sigma}\right)$ as

$$
\begin{gathered}
n^{\sigma}(t)=G(t) *\left(n_{0} * \rho^{\sigma}\right)-\int_{0}^{t} \nabla G(t-s) *\left(n^{\sigma}(s) \nabla c^{\sigma}(s)\right) d s, \\
c^{\sigma}(t)=e^{-\alpha t / \varepsilon} G(t / \varepsilon) *\left(c_{0} * \rho^{\sigma}\right)+\frac{1}{\varepsilon} \int_{0}^{t / \varepsilon} e^{\alpha(s-t) / \varepsilon} G((t-s) / \varepsilon) *\left(n^{\sigma}(s / \varepsilon) * \rho^{\sigma}\right) d s,
\end{gathered}
$$

with $G(x, t)=\frac{1}{4 \pi t} e^{-|x|^{2} /(4 t)}$ the heat kernel in $\mathbb{R}^{2}$, because the nonlinearity is Lipschitz (see [2]). The smoothness of $\left(n^{\sigma}, c^{\sigma}\right)$ follows by the regularizing property of the heat equation and by the smoothness of the initial data. The non-negativity of $\left(n^{\sigma}, c^{\sigma}\right)$ follows by the maximum principle. As a consequence, the a priori estimates in Theorem 3.2 hold true for the regularized solution $\left(n^{\sigma}, c^{\sigma}\right)$. These a priori bounds give a global in time control of the energy and energy dissipation, still uniform in $\sigma$. This gives space compactness on $c$ and $\nabla n$. The Lions-Aubin compactness method gives the required time compactness.

\section{Blow-up}

In this section we shall prove Theorem 1.2, which states a blow-up result for the parabolic-elliptic system (1.1) and for super-critical mass, i.e. $M>8 \pi$. Since the same result in the case $\varepsilon=\alpha=0$ has been given in [6], we assume $\alpha>0$ so that $c=B_{\alpha} * n$. The result in [6] will be recovered in the limit $\alpha \rightarrow 0$.

Let us denote $I(t)=\int_{\mathbb{R}^{2}}|x|^{2} n(x, t) d x$. Then, $I(t)$ satisfies the differential equation

$$
\frac{d}{d t} I(t)=4 M+2 \int_{\mathbb{R}^{2}} n(x, t) x \cdot\left(\nabla B_{\alpha} * n(t)\right)(x) d t .
$$

Using the computation

$$
\nabla B_{\alpha}(z)=-\frac{z}{8 \pi} \int_{0}^{+\infty} \frac{1}{t^{2}} e^{-\frac{|z|^{2}}{4 t}-\alpha t} d t=-\frac{1}{2 \pi} \frac{z}{|z|^{2}} \int_{0}^{+\infty} e^{-s-\alpha \frac{|z|^{2}}{4 s}} d s
$$


and denoting $g_{\alpha}(z)=\int_{0}^{+\infty} e^{-s-\alpha \frac{|z|^{2}}{4 s}} d s$, we obtain

$$
\begin{aligned}
\frac{d}{d t} I(t) & =4 M-\frac{1}{\pi} \iint_{\mathbb{R}^{2} \times \mathbb{R}^{2}} n(x, t) \frac{x \cdot(x-y)}{|x-y|^{2}} g_{\alpha}(x-y) n(y, t) d y d x \\
& =4 M-\frac{1}{2 \pi} \iint_{\mathbb{R}^{2} \times \mathbb{R}^{2}} n(x, t) g_{\alpha}(x-y) n(y, t) d y d x
\end{aligned}
$$

Since $g_{\alpha}$ is a positive radial decreasing function such that $g_{\alpha}(z) \leq 1$ and $g_{\alpha}(z) \rightarrow 1$ as $\alpha \rightarrow 0$ for all $z \in \mathbb{R}^{2}$, we recover from (7.1) the result in [6]. For $\alpha>0$ we have

$$
\frac{d}{d t} I(t)=4 M\left(1-\frac{M}{8 \pi}\right)+\frac{1}{2 \pi} \int_{\mathbb{R}^{2}} \int_{\mathbb{R}^{2}} n(x, t)\left[1-g_{\alpha}(x-y)\right] n(y, t) d y d x,
$$

and one has to estimate the second term in the right hand side of (7.2) in term of $I(t)$. Denoting $r=|z|$, we observe that

$$
\frac{d}{d r}\left(1-g_{\alpha}(r)\right)=\frac{\alpha}{2} r \int_{0}^{+\infty} \frac{1}{s} e^{-s-\alpha \frac{r^{2}}{4 s}} d s=2 \pi \alpha r B_{1}(\sqrt{\alpha} r) .
$$

Then, reasoning as in Lemma 4.2, we deduce from (7.3) that

$$
\frac{d}{d r}\left(1-g_{\alpha}(r)\right) \leq \sqrt{\alpha} K, \quad \forall 0<r<\frac{1}{\sqrt{\alpha}}
$$

where $K=2 \pi \sup _{\rho \in(0,1)} \rho B_{1}(\rho)<+\infty$. As a consequence of $(7.4)$ and since $0 \leq 1-$ $g_{\alpha}(z) \leq 1$ for all $z \in \mathbb{R}^{2}$, we obtain the estimate

$$
0 \leq 1-g_{\alpha}(z) \leq \sqrt{\alpha} \mathcal{C}|z|, \quad \forall z \in \mathbb{R}^{2}
$$

with $\mathcal{C}=\max \{K, 1\}$.

Finally, inserting (7.5) into (7.2), we get

$$
\begin{aligned}
\frac{d}{d t} I(t) & \leq 4 M\left(1-\frac{M}{8 \pi}\right)+\frac{\sqrt{\alpha}}{2 \pi} \mathcal{C} \iint_{\mathbb{R}^{2} \times \mathbb{R}^{2}} n(x, t)|x-y| n(y, t) d y d x \\
& \leq 4 M\left(1-\frac{M}{8 \pi}\right)+\frac{\sqrt{\alpha}}{\pi} \mathcal{C} M \int_{\mathbb{R}^{2}}|x| n(x, t) d x \\
& \leq 4 M\left(1-\frac{M}{8 \pi}\right)+\frac{\sqrt{\alpha}}{\pi} \mathcal{C} M^{3 / 2} \sqrt{I(t)}
\end{aligned}
$$

i.e.,

$$
I(t) \leq I(0)+\int_{0}^{t} f(I(s)) d s
$$

where $f(\lambda)=\frac{M}{2 \pi}(8 \pi-M)+\frac{\sqrt{\alpha}}{\pi} \mathcal{C} M^{3 / 2} \lambda^{\frac{1}{2}}$. Since $f$ is a strictly increasing function such that $f\left(\lambda^{*}\right)=0$ for $\lambda^{*}=\frac{1}{4 \alpha \mathcal{C}^{2} M}(M-8 \pi)^{2}$, the hypothesis $(1.2)$ gives us $I(0)<\lambda^{*}$ and $f(I(0))<0$. Therefore, $\int_{0}^{t} f(I(s)) d s<0$ as soon as (7.6) holds true and $I(t) \leq$ $I(0)+t f(I(0))$ as soon as (7.6) holds true, i.e., the second momentum becomes necessarily non-positive for $t \geq-\frac{I(0)}{f(I(0))}=\frac{2 \pi I_{0}}{M\left(M-8 \pi-2 \mathcal{C} \sqrt{\alpha M I_{0}}\right)}$, expressing in this way the formation of a singularity before. 


\section{Appendix A.}

A.1. The entropy minimization lemma. The entropy minimization Lemma 2.1 is very classical and several proofs are available. For example, we may use the Jensen's inequality with respect to the probability measure $\frac{n}{M} d x$, and we deduce

$$
\begin{aligned}
\frac{1}{M} \int_{\mathbb{R}^{2}}(n(x) \log n(x)-n(x) \psi(x)) d x & =-\int_{\mathbb{R}^{2}} \log \left(\frac{e^{\psi(x)}}{n(x)}\right) \frac{n(x)}{M} d x \\
& \geq-\log \left(\frac{1}{M} \int_{\mathbb{R}^{2}} e^{\psi(x)} d x\right)=\frac{1}{M} E(\bar{n} ; \psi) .
\end{aligned}
$$

One can also invoke the Legendre transform of the functional $\int_{\mathbb{R}^{2}} n(x) \log n(x) d x$ to obtain (2.11). However, in these ways we lose the identity in (2.11). Therefore, the following proof, in the line of [9], is more complete.

Proof. [Proof of the entropy minimization Lemma 2.1.] First of all, by the definition of $\bar{n}$ we have that $\bar{n} \in \mathcal{U}$ and

$$
\log \bar{n}=\psi+\log \left(M / \int_{\mathbb{R}^{2}} e^{\psi} d x\right) .
$$

Therefore, the entropy functional $E$ is finite in $\bar{n}$ and it takes the value $E(\bar{n} ; \psi)=$ $M \log \left(M / \int_{\mathbb{R}^{2}} e^{\psi} d x\right)$. Next, it is easy to see that for any $n$ in $\mathcal{U}, E(n ; \psi)$ and $R E(n \mid \bar{n})$ are finite or infinite in the same time and that (2.11) holds true. Indeed, from (A.1) we deduce $n \log (n / \bar{n})=n \log n-n \psi-n \log \left(M / \int_{\mathbb{R}^{2}} e^{\psi} d x\right)$. The non-negativity of $R E(n \mid \bar{n})$ over the set $\mathcal{U}$ follows by the computation

$$
R E(n \mid \bar{n})=\int_{\mathbb{R}^{2}}(n(x) \log n(x)-\bar{n}(x) \log \bar{n}(x)-(\log \bar{n}(x)+1)(n(x)-\bar{n}(x))) d x,
$$

and the convexity of the function $u \mapsto u \log u$.

\section{A.2. The chemical energy minimization lemma.}

Proof. [Proof of the chemical energy minimization Lemma 2.2.] Let us start first with the basic regularity properties of $\bar{c}$ (see[22]). If $\alpha>0$, then $\bar{c} \in L^{p}\left(\mathbb{R}^{2}\right)$ for all $p \in$ $[1, \infty)$, since $f \in L^{1}\left(\mathbb{R}^{2}\right)$ by hypothesis. On the other hand, when $\alpha=0, \bar{c} \in L_{\text {loc }}^{1}\left(\mathbb{R}^{2}\right)$, since $f \in L^{1}\left(\mathbb{R}^{2}\right) \cap L^{1}\left(\mathbb{R}^{2}, \log \left(1+|x|^{2}\right) d x\right)$. Moreover (2.13) holds true in $\mathcal{D}^{\prime}\left(\mathbb{R}^{2}\right)$ as well as $-\Delta \bar{c}+\alpha \bar{c}=f$ for $\alpha \geq 0$.

Next we aim to justify the integration by parts arising in the computation (2.14). Let us assume that we know a priori that $|\nabla \bar{c}| \in L^{2}\left(\mathbb{R}^{2}\right)$. Then $c \Delta \bar{c} \in L^{1}\left(\mathbb{R}^{2}\right)$ for all $c \in H^{1}\left(\mathbb{R}^{2}\right)$, and the following partial integration holds true:

$$
\int_{\mathbb{R}^{2}} \nabla c(x) \cdot \nabla \bar{c}(x) d x=-\int_{\mathbb{R}^{2}} c(x) \Delta \bar{c}(x) d x=\int_{\mathbb{R}^{2}} c(x)(f(x)-\alpha \bar{c}(x)) d x .
$$

Indeed, thanks to the hypothesis and the basic properties of $\bar{c}$, we can decompose $-\Delta \bar{c}=g_{1}+g_{2}$, with $g_{1} \geq 0$ in $L_{l o c}^{1}\left(\mathbb{R}^{2}\right)$ defined as

$$
g_{1}:=f+\alpha(\bar{c})_{-} \quad \text { if } \alpha>0 \text { and } \quad g_{1}:=f_{1} \quad \text { if } \alpha=0
$$

and $g_{2} \in L^{2}\left(\mathbb{R}^{2}\right)$ defined as

$$
g_{2}:=-\alpha(\bar{c})_{+} \quad \text { if } \alpha>0 \text { and } \quad g_{2}:=f_{2} \quad \text { if } \alpha=0
$$


(see [22, Theorem 7.7]). Moreover, $F_{\alpha}(c ; f)$ is finite.

As a consequence of (A.2), we obtain (2.14), since

$$
\begin{aligned}
\frac{1}{2} \int_{\mathbb{R}^{2}}|\nabla c-\nabla \bar{c}|^{2}+\frac{\alpha}{2} \int_{\mathbb{R}^{2}}(c-\bar{c})^{2} d x & =F_{\alpha}(c ; f)+\frac{1}{2} \int_{\mathbb{R}^{2}}|\nabla \bar{c}|^{2} d x+\frac{\alpha}{2} \int_{\mathbb{R}^{2}} \bar{c}^{2} d x \\
& =F_{\alpha}(c ; f)-F_{\alpha}(\bar{c} ; f) .
\end{aligned}
$$

It remains to prove that $|\nabla \bar{c}| \in L^{2}\left(\mathbb{R}^{2}\right)$. Let us consider first the case $\alpha=0$, which is much more complicated than the case $\alpha>0$, since the fundamental solution $E_{2}$ does not lie in any $L^{p}\left(\mathbb{R}^{2}\right)$ spaces and $\nabla \bar{c}=\nabla E_{2} * f$ in general does not lie in $L^{2}\left(\mathbb{R}^{2}\right)$ because of the critical fractional Sobolev embedding (see [22]). The case $\alpha>0$ is considered subsequently.

In the case $\alpha=0$, the hypothesis $\int_{\mathbb{R}^{2}} f(x) d x=0$ allows us to subtract any function of the variable $x$ inside the convolution formulation for $\nabla \bar{c}$. Let us consider two radii $0<r<1<R$ with $R>e-1$, and let us denote $\mathcal{C} B(0, r)=\mathbb{R}^{2} \backslash B(0, r)$. Then we split $\nabla \bar{c}$ in the following way:

$$
\begin{aligned}
\nabla \bar{c}(x)= & -\frac{1}{2 \pi} \int_{\mathbb{R}^{2}}\left(\frac{x-y}{|x-y|^{2}}-\frac{x}{1+|x|^{2}} \mathbf{1}_{\mathcal{C} B(0, R)}(x)\right) f(y) d y \\
= & -\frac{1}{2 \pi} \int_{\mathbb{R}^{2}}\left(\frac{x-y}{|x-y|^{2}}-\frac{x}{1+|x|^{2}} \mathbf{1}_{\mathcal{C} B(0, R)}(x)\right) \mathbf{1}_{B(0, r)}(x-y) f(y) d y \\
& -\frac{1}{2 \pi} \int_{\mathbb{R}^{2}}\left(\frac{x-y}{|x-y|^{2}}-\frac{x}{1+|x|^{2}} \mathbf{1}_{\mathcal{C} B(0, R)}(x)\right) \mathbf{1}_{\mathcal{C} B(0, r)}(x-y) f(y) d y \\
= & : I_{1}(x)+I_{2}(x)
\end{aligned}
$$

and we will show separately that $\left|I_{1}\right|$ and $\left|I_{2}\right|$ lie in $L^{2}\left(\mathbb{R}^{2}\right)$.

Concerning $I_{1}$, we have for all $x \in \mathbb{R}^{2}$ that

$$
\begin{aligned}
& \left|I_{1}(x)\right| \leq \frac{1}{2 \pi} \int_{\mathbb{R}^{2}} \frac{1}{|x-y|} \mathbf{1}_{B(0, r)}(x-y)|f(y)| d y \\
& \quad+\frac{|x| \mathbf{1}_{\mathcal{C} B(0, R)}(x)}{2 \pi\left(1+|x|^{2}\right)} \int_{\mathbb{R}^{2}} \mathbf{1}_{B(0, r)}(x-y)|f(y)| d y .
\end{aligned}
$$

Denoting $\Omega_{x}=\left\{y \in \mathbb{R}^{2}: f_{1}(y)>\frac{1}{|x-y|}\right\}$, the first integral in the right hand side of (A.4) can be split again in the following way:

$$
\begin{aligned}
\int_{\mathbb{R}^{2}} \frac{1}{|x-y|} \mathbf{1}_{B(0, r)}(x-y)|f(y)| d y \leq & \int_{\mathbb{R}^{2}} \frac{1}{|x-y|} \mathbf{1}_{B(0, r)}(x-y) f_{1}(y) \mathbf{1}_{\Omega_{x}}(y) d y \\
& +\int_{\mathbb{R}^{2}} \frac{1}{|x-y|} \mathbf{1}_{B(0, r)}(x-y) f_{1}(y) \mathbf{1}_{\mathcal{C} \Omega_{x}}(y) d y \\
& +\int_{\mathbb{R}^{2}} \frac{1}{|x-y|} \mathbf{1}_{B(0, r)}(x-y)\left|f_{2}(y)\right| d y \\
= & : I_{1,1}(x)+I_{1,2}(x)+I_{1,3}(x) .
\end{aligned}
$$

Since for $y \in B(x, r) \cap \Omega_{x}$ it holds true that $f_{1}(y)>\frac{1}{|x-y|}>\frac{1}{r}>1$, we have that

$$
0 \leq I_{1,1}(x) \leq \int_{\mathbb{R}^{2}} \frac{1}{|x-y|} \frac{1}{\log \left(\frac{1}{|x-y|}\right)} \mathbf{1}_{B(0, r)}(x-y) f_{1}(y) \log f_{1}(y) \mathbf{1}_{\Omega_{x}}(y) d y
$$


Therefore, since the right hand side of (A.6) belongs to $L^{2}\left(\mathbb{R}^{2}\right)$ thanks to Young's inequality, $I_{1,1} \in L^{2}\left(\mathbb{R}^{2}\right)$ too. On the other hand, since for $y \in B(x, r) \cap \mathcal{C} \Omega_{x}$ it holds true that $f_{1}(y) \leq \frac{1}{|x-y|}$, we have

$$
0 \leq I_{1,2}(x) \leq \int_{\mathbb{R}^{2}} \frac{1}{|x-y|^{3 / 2}} \mathbf{1}_{B(0, r)}(x-y) \sqrt{f_{1}(y)} \mathbf{1}_{\mathcal{C} \Omega_{x}}(y) d y .
$$

Again by Young's inequality the right hand side of (A.7) belongs to $L^{2}\left(\mathbb{R}^{2}\right)$ and so $I_{1,2}$ too. The term $I_{1,3}$ in (A.5) belongs to $L^{2}\left(\mathbb{R}^{2}\right)$ by Young's inequality, since $f_{2} \in$ $L^{2}\left(\mathbb{R}^{2}\right)$. Finally, since for $x \in \mathcal{C} B(0, R)$ and $y \in B(x, r)$ we have $|y| \geq|x|-r \geq R-r>0$, the second term in the right hand side of (A.4) can be dominated in the following way:

$$
\begin{aligned}
& \frac{|x| \mathbf{1}_{\mathcal{C} B(0, R)}(x)}{1+|x|^{2}} \int_{\mathbb{R}^{2}} \mathbf{1}_{B(0, r)}(x-y)|f(y)| d y \\
& \quad \leq \frac{|x| \mathbf{1}_{\mathcal{C} B(0, R)}(x)}{\left(1+|x|^{2}\right) \log \left(1+(|x|-r)^{2}\right)} \int_{\mathbb{R}^{2}}|f(y)| \log \left(1+|y|^{2}\right) d y .
\end{aligned}
$$

Therefore, it belongs to $L^{2}\left(\mathbb{R}^{2}\right)$. Collecting (A.4), (A.5), (A.6), (A.7) and (A.8), we obtain that $\left|I_{1}\right|$ belongs to $L^{2}\left(\mathbb{R}^{2}\right)$.

Concerning $I_{2}$, it is enough to prove that $\left|I_{2}\right| \in L^{2}(\mathcal{C} B(0, R))$ since $\left|I_{2}\right| \in L^{\infty}\left(\mathbb{R}^{2}\right)$. Let $x \in \mathcal{C} B(0, R)$ and let us define $\Omega_{x}^{\prime}=\left\{y \in \mathbb{R}^{2}:|y|<|x| / \log (1+|x|)\right\}$. Then,

$$
\begin{aligned}
\left|I_{2}(x)\right| \leq & \frac{1}{2 \pi} \int_{\mathbb{R}^{2}}\left|\frac{x-y}{|x-y|^{2}}-\frac{x}{1+|x|^{2}}\right| \mathbf{1}_{\mathcal{C} B(0, r)}(x-y)|f(y)| \mathbf{1}_{\Omega_{x}^{\prime}}(y) d y \\
& \quad+\frac{1}{2 \pi} \int_{\mathbb{R}^{2}}\left|\frac{x-y}{|x-y|^{2}}-\frac{x}{1+|x|^{2}}\right| \mathbf{1}_{\mathcal{C} B(0, r)}(x-y)|f(y)| \mathbf{1}_{\mathcal{C} \Omega_{x}^{\prime}}(y) d y \\
= & : I_{2,1}(x)+I_{2,2}(x) .
\end{aligned}
$$

For $x \in \mathcal{C} B(0, R)$ and $y \in \Omega_{x}^{\prime}$ it holds true that $|x|-|y|>|x|\left(1-\frac{1}{\log (1+|x|)}\right)>0$ since $R>e-1$, and

$$
\begin{aligned}
\left|\frac{x-y}{|x-y|^{2}}-\frac{x}{1+|x|^{2}}\right| & \leq \frac{\left|(x-y)\left(1+|x|^{2}\right)-x\left(|x|^{2}+|y|^{2}-2 x \cdot y\right)\right|}{(|x|-|y|)^{2}\left(1+|x|^{2}\right)} \\
& \leq \frac{|x|+|y|+3|y||x|^{2}+|x||y|^{2}}{\left(1+|x|^{2}\right)} \frac{\log ^{2}(1+|x|)}{|x|^{2}(\log (1+|x|)-1)^{2}} \\
& \leq C \frac{\log (1+|x|)}{|x|(\log (1+|x|)-1)^{2}}=: h(x) .
\end{aligned}
$$

Therefore, $\left|I_{2,1}(x)\right| \leq \frac{1}{2 \pi}\|f\|_{L^{1}\left(\mathbb{R}^{2}\right)} h(x)$, which implies that $I_{2,1} \in L^{2}(\mathcal{C} B(0, R))$. Finally,

$$
\begin{aligned}
0 \leq I_{2,2}(x) \leq \frac{1}{2 \pi} \int_{\mathbb{R}^{2}} \frac{1}{|x-y|} \mathbf{1}_{\mathcal{C} B(0, r)}(x-y)|f(y)| \mathbf{1}_{\mathcal{C} \Omega_{x}^{\prime}}(y) d y \\
\quad+\frac{|x|}{2 \pi\left(1+|x|^{2}\right)} \int_{\mathbb{R}^{2}} \mathbf{1}_{\mathcal{C} B(0, r)}(x-y)|f(y)| \mathbf{1}_{\mathcal{C} \Omega_{x}^{\prime}}(y) d y
\end{aligned}
$$


The second integral in the right hand side of $(\mathrm{A} .11)$ belongs to $L^{2}(\mathcal{C} B(0, R))$, because $|y| \geq \frac{|x|}{\log (1+|x|)}$ for $y \in \mathcal{C} \Omega_{x}^{\prime}$ so that

$$
\begin{aligned}
& \frac{|x|}{\left(1+|x|^{2}\right)} \int_{\mathbb{R}^{2}} \mathbf{1}_{\mathcal{C} B(0, r)}(x-y)|f(y)| \mathbf{1}_{\mathcal{C} \Omega_{x}^{\prime}}(y) d y \\
& \quad \leq \frac{|x|}{\left(1+|x|^{2}\right) \log \left(1+\frac{|x|^{2}}{\log ^{2}(1+|x|)}\right)} \int_{\mathbb{R}^{2}} \log \left(1+|y|^{2}\right)|f(y)| d y \in L^{2}(\mathcal{C} B(0, R)) .
\end{aligned}
$$

In order to control the first integral in the right hand side of (A.11), we observe that for all $x \in \mathcal{C} B(0, R)$ and $y \in \mathcal{C} \Omega_{x}^{\prime}$ it holds true that

$$
\begin{aligned}
\log \left(1+\frac{\frac{1}{2}|x-y|}{\log \left(1+\frac{1}{2}|x-y|\right)}\right) & \leq \max \left(\log \left(1+\frac{|x|}{\log (1+|x|)}\right) ; \log \left(1+\frac{|y|}{\log (1+|y|)}\right)\right) \\
& \leq \log \left(1+|y|^{2}\right) .
\end{aligned}
$$

Indeed, the function $\varphi(s)=\frac{s}{\log (1+s)}$ is strictly increasing for $s>-1$ such that $\varphi(e-$ $1)=e-1$. Then using the inequality $\frac{1}{2}|x-y| \leq \max (|x| ;|y|)$, the first inequality (A.13) follows. Moreover, for $x \in \mathcal{C} B(0, R)$ and $y \in \mathcal{C} \Omega_{x}^{\prime}$ we have

$$
e-1 \leq \frac{|x|}{\log (1+|x|)} \leq|y| \text { and } \frac{|y|}{\log (1+|y|)} \leq|y| \leq|y|^{2},
$$

and the second inequality (A.14) follows too. Therefore,

$$
\begin{aligned}
& \int_{\mathbb{R}^{2}} \frac{1}{|x-y|} \mathbf{1}_{\mathcal{C} B(0, r)}(x-y)|f(y)| \mathbf{1}_{\mathcal{C} \Omega_{x}^{\prime}}(y) d y \\
\leq & \int_{\mathbb{R}^{2}} \frac{1}{|x-y|} \frac{1}{\log \left(1+\frac{\frac{1}{2}|x-y|}{\log \left(1+\frac{1}{2}|x-y|\right)}\right)} \mathbf{1}_{\mathcal{C} B(0, r)}(x-y) \log \left(1+|y|^{2}\right)|f(y)| \mathbf{1}_{\mathcal{C} \Omega_{x}^{\prime}}(y) d y .
\end{aligned}
$$

Collecting (A.9), (A.10), (A.11), (A.12) and (A.15) we have proved that $\left|I_{2}\right| \in$ $L^{2}(\mathcal{C} B(0, R))$, and the case $\alpha=0$ is solved.

When $\alpha>0$, we have that $\left|\nabla B_{\alpha}(z)\right|=\frac{1}{2 \pi} \frac{1}{|z|} g_{\alpha}(z)$ with $g_{\alpha}(z)=\int_{0}^{+\infty} e^{-s-\alpha \frac{|z|^{2}}{4 s}} d s$, (see Section 7 ). Therefore, $\left|\nabla B_{\alpha}(z)\right|$ has the same singularity as $\left|\nabla E_{2}(z)\right|$ in $z=0$, but $\left|\nabla B_{\alpha}(z)\right| \rightarrow 0$ exponentially as $|z| \rightarrow+\infty$. As a consequence, we can prove that $\nabla \bar{c}=$ $\nabla B_{\alpha} * f \in L^{2}\left(\mathbb{R}^{2}\right)$ using the previous technique and without subtracting any function of the variable $x$ inside the convolution formulation for $\nabla \bar{c}$.

A.3. A remark on the case $\varepsilon=\alpha=0$. In the case of the parabolic-elliptic system (1.1) with $\varepsilon=\alpha=0$ and

$$
c(x, t)=-\frac{1}{2 \pi} \int_{\mathbb{R}^{2}} \log |x-y| n(y, t) d y,
$$

as far as global existence is concerned, it is sufficient to assume that the cell density $n_{0}$ satifies both

$$
\int_{\mathbb{R}^{2}} n_{0}(x) \log n_{0}(x) d x<\infty \quad \text { and } \quad \int_{\mathbb{R}^{2}} n_{0}(x) \log \left(1+|x|^{2}\right) d x<\infty .
$$


In fact, assumptions (A.16) are the optimal ones for several viewpoints. First, they are minimal for applying the logarithmic HLS inequality (4.1). Furthermore, the combination of these two ensures that the mass does not escape to infinity by Lemma 2.4 and the balance is optimal again. Last but not least, it can be proved using free energy methods that conditions (A.16) are indeed propagated along the solutions, with local in time bounds.

Following the lines of computation (3.4) for instance, we have to estimate $\int_{\mathbb{R}^{2}} c(x, t) H(x) d x$ from above. This can be done with the following calculation,

$$
\begin{aligned}
\int_{\mathbb{R}^{2}} c(x, t) H(x) d x & =-\frac{1}{2 \pi} \iint_{\mathbb{R}^{2} \times \mathbb{R}^{2}} n(y, t) \log |x-y| H(x) d y d x \\
& =\frac{1}{8 \pi} \int_{\mathbb{R}^{2}} n(y, t) \log H(y) d y+C,
\end{aligned}
$$

which can be viewed as an integration by parts knowing (3.8) (see also [8], where the Euler-Lagrange equation for $H$ is clearly noticed). The latter is then trivially bounded from above, because $\int_{\mathbb{R}^{2}} n(y, t) \log H(y) d y$ is nonpositive.

Concerning the blow-up of solutions, the assumption on the second momentum, $\int_{\mathbb{R}^{2}}|x|^{2} n_{0}(x) d x<+\infty$, seems, however, to be crucial. For instance, for the critical mass value $M=8 \pi$, there exists a family of stationary states with infinite second momentum but finite "logarithmic" momentum, for which blow-up obviously does not occur (see $[3,5])$.

A.4. The duality. There is a true duality between the Onofri and the logarithmic Hardy-Littlewood-Sobolev inequalities (see [8, 4]). We followed this idea all along this paper. In this appendix we give a formal proof of this duality in the whole space $\mathbb{R}^{2}$ for the sake of completeness, and we then derive another proof of Lemma 4.2.

First of all, we write the Onofri's inequality as

$$
\frac{1}{8 \pi} \log \left(\int_{\mathbb{R}^{2}} e^{8 \pi u(x)} H(x) d x\right) \leq \int_{\mathbb{R}^{2}} u(x) H(x) d x+\frac{1}{2} \int_{\mathbb{R}^{2}}|\nabla u(x)|^{2} d x .
$$

Let $f$ be a function satisfying the hypotheses of Lemma 4.1 and $\int_{\mathbb{R}^{2}} f(x) d x=1$ without loss of generality. By the minimization procedure (see Lemma 2.2 and Appendix A.2) we have

$$
\begin{aligned}
& -\frac{1}{2} \int_{\mathbb{R}^{2}}(f-H)(x)\left(E_{2} *(f-H)\right)(x) d x \\
& \quad=\min _{u}\left\{\frac{1}{2} \int_{\mathbb{R}^{2}}|\nabla u(x)|^{2} d x-\int_{\mathbb{R}^{2}}(f-H)(x) u(x) d x\right\} \\
& \quad \geq \min _{u}\left\{\frac{1}{8 \pi} \log \left(\int_{\mathbb{R}^{2}} e^{8 \pi u(x)} H(x) d x\right)-\int_{\mathbb{R}^{2}} f(x) u(x) d x\right\} \\
& \quad \geq \min _{u}\left\{-\frac{1}{8 \pi} \int_{\mathbb{R}^{2}} f(x) \log f(x)-f(x)(8 \pi u(x)+\log H(x)) d x-\int_{\mathbb{R}^{2}} f(x) u(x) d x\right\} \\
& \quad \geq-\frac{1}{8 \pi} \int_{\mathbb{R}^{2}} f(x) \log f(x) d x+\frac{1}{8 \pi} \int_{\mathbb{R}^{2}} f(x) \log H(x) d x,
\end{aligned}
$$

from the entropy minimization Lemma 2.1 (see Appendix A.1). On the other hand, the Euler-Lagrange formula for $H$ writes [8]

$$
-\int_{\mathbb{R}^{2}} \log |x-y| H(y) d y=\frac{1}{4} \log H(x)+\frac{1}{4} \int_{\mathbb{R}^{2}} H(x) \log H(x) d x+C_{0} .
$$


It is in fact the dual formulation of the cancellation property (3.8). We deduce that

$$
\begin{gathered}
\frac{1}{4 \pi} \iint_{\mathbb{R}^{2} \times \mathbb{R}^{2}} f(x) \log |x-y| f(y) d x d y+\frac{1}{4 \pi} \iint_{\mathbb{R}^{2} \times \mathbb{R}^{2}} H(x) \log |x-y| H(y) d x d y \\
\geq \frac{1}{2 \pi} \iint_{\mathbb{R}^{2} \times \mathbb{R}^{2}} f(x) \log |x-y| H(y) d x d y-\frac{1}{8 \pi} \int_{\mathbb{R}^{2}} f(x) \log f(x) d x \\
\quad+\frac{1}{8 \pi} \int_{\mathbb{R}^{2}} f(x) \log H(x) d x \\
\geq-\frac{1}{8 \pi} \int_{\mathbb{R}^{2}} f(x) \log f(x) d x-\frac{1}{8 \pi} \int_{\mathbb{R}^{2}} H(x) \log H(x) d x+\frac{C_{0}}{2 \pi} .
\end{gathered}
$$

We have recovered the logarithmic Hardy-Littlewood-Sobolev inequality with the sharp constant.

Following the previous lines there is another way to derive the modified inequality for the kernel $B_{\alpha}$ (Lemma 4.2). We proceed as above, more directly because the Bessel kernel has nicer properties at infinity:

$$
\begin{aligned}
& -\frac{1}{2} \int_{\mathbb{R}^{2}} f(x)\left(B_{\alpha} * f\right)(x) d x \\
= & \min _{u}\left\{\frac{1}{2} \int_{\mathbb{R}^{2}}|\nabla u(x)|^{2} d x+\frac{\alpha}{2} \int_{\mathbb{R}^{2}} u^{2}(x) d x-\int_{\mathbb{R}^{2}} f(x) u(x) d x\right\} \\
\geq & \min _{u}\left\{\frac{1}{8 \pi} \log \left(\int_{\mathbb{R}^{2}} e^{8 \pi u(x)} H(x) d x\right)-\int_{\mathbb{R}^{2}} f(x) u(x) d x\right. \\
\left.+\frac{\alpha}{2} \int_{\mathbb{R}^{2}} u^{2}(x) d x-\int_{\mathbb{R}^{2}} u(x) H(x) d x\right\} & \\
\geq \min _{u}\left\{-\frac{1}{8 \pi} \int_{\mathbb{R}^{2}} f(x) \log f(x)-f(x)(8 \pi u(x)+\log H(x)) d x-\int_{\mathbb{R}^{2}} f(x) u(x) d x\right. & \left.\quad+\frac{\alpha}{2} \int_{\mathbb{R}^{2}} u^{2}(x) d x-\int_{\mathbb{R}^{2}} u(x) H(x) d x\right\} \\
\geq & -\frac{1}{8 \pi} \int_{\mathbb{R}^{2}} f(x) \log f(x) d x+\frac{1}{8 \pi} \int_{\mathbb{R}^{2}} f(x) \log H(x) d x+C(\alpha),
\end{aligned}
$$

because we have for $\alpha>0$ :

$$
\int_{\mathbb{R}^{2}} u(x) H(x) d x \leq \frac{\alpha}{2} \int_{\mathbb{R}^{2}} u^{2}(x) d x+\frac{1}{2 \alpha} \int_{\mathbb{R}^{2}} H^{2}(x) d x .
$$

To conclude this Appendix let us mention that there exists a third strategy to prove Lemma 4.2, which is based on a "weak logarithmic HLS inequality" (see [4, Theorem 3]).

\section{REFERENCES}

[1] A. Arnold, P. Markowich, G. Toscani and A. Unterreiter, On convex Sobolev inequalities and the rate of convergence to equilibrium for Fokker-Planck type equations, Comm. P.D.E., 26, 43-100, 2001.

[2] P. Biler, Local and global solvability of some parabolic systems modelling chemotaxis, Adv. Math. Sci. Appl., 8, 715-743, 1998.

[3] P. Biler, G. Karch, P. Laurençot and T. Nadzieja, The $8 \pi$-problem for radially symmetric solutions of a chemotaxis model in the plane, Math. Methods Appl. Sci., 29, 1563-1583, 2006 . 
[4] W. Beckner, Sharp Sobolev inequalities on the sphere and the Moser-Trudinger inequality, Ann. of Math., 138, 213-242, 1993.

[5] A. Blanchet, J.A. Carrillo and N. Masmoudi, Infinite time aggregation for the critical PatlakKeller-Segel model in $\mathbb{R}^{2}$, Comm. Pure Appl. Math., to appear, 2007.

[6] A. Blanchet, J. Dolbeault and B. Perthame, Two-dimensional Keller-Segel model: optimal critical mass and qualitative properties of the solutions, Electron. J. Diff. Eqns., 44, 1-33, 2006.

[7] V. Calvez and J.A. Carrillo, Volume effects in the Keller-Segel model: energy estimates preventing blow-up, J. Math. Pures Appl., 86, 155-175, 2006.

[8] E. Carlen and M. Loss, Competing symmetries, the logarithmic HLS inequality and Onofri's inequality on $\mathbb{S}^{n}$, Geom. Funct. Anal., 2, 90-104, 1992.

[9] J.A. Carrillo, A. Jungel, P.A. Markowich, G. Toscani and A. Unterreiter, Entropy dissipation methods for degenerate parabolic problems and generalized Sobolev inequalities, Monatsh. Math., 133, 1-82, 2001.

[10] F. Chalub, Y. Dolak-Struss, P. Markowich, D. Oelz, C. Schmeiser and A. Soreff, Model hierarchies for cell aggregation by chemotaxis, Math. Models Methods Appl. Sci., 16, 1173-1197, 2006.

[11] L. Corrias and B. Perthame, Critical space for the parabolic-parabolic Keller-Segel model in $\mathbb{R}^{d}$, C. R. Acad. Sci. Paris, Ser. I, 342, 745-750, 2006.

[12] L. Corrias and B. Perthame, Asymptotic decay for the solutions of the parabolicparabolic Keller-Segel chemotaxis system in critical spaces, Math. Comp. Model., 2007. doi:10.1016/j.mcm.2007.06.005.

[13] L. Corrias, B. Perthame and H. Zaag, Global solutions of some chemotaxis and angiogenesis systems in high space dimensions, Milano J. of Math., 72, 1-29, 2004.

[14] W.F. Donoghue, Distributions and Fourier Transforms, Pure and Applied Mathemathics, Academic Press, 32, 1969.

[15] R. Erban and H.G. Othmer, Taxis equations for amoeboid cells, J. Math. Biol., 54, 847-885, 2007.

[16] H. Gajewski and K. Zacharias, Global behavior of a reaction-diffusion system modelling chemotaxis, Math. Nachr., 195, 77-114, 1998.

[17] M.A. Herrero and J.L.L Velázquez, Chemotactic collapse for the Keller-Segel model, J. Math. Biol., 35, 177-194, 1996.

[18] T. Höfer, J.A. Sherratt and P.K. Maini, Dictyostelium discoideum: cellular self-organisation in an excitable medium, Proc. Roy. Soc. Lond. B, 259, 249-257, 1995.

[19] W. Jäger and S. Luckhaus, On explosions of solutions to a system of partial differential equations modeling chemotaxis, Trans. Amer. Math. Soc., 239, 819-821, 1992.

[20] H. Khonsari and V. Calvez, The origins of concentric demyelination: self-organization in the human brain, PLoS ONE, 2(1), e150, 2007. doi:10.1371/journal.pone.0000150.

[21] E.F. Keller and L.A. Segel, Initiation of slime mold aggregation viewed as an instability, J. Theor. Biol., 26, 399-415, 1970.

[22] E.H. Lieb and M. Loss, Graduate Studies in Mathematics, Analysis, second edition, American Mathematical Society, 14, 2001.

[23] T. Nagai, Global existence and blow-up of solutions to a chemotaxis system, Nonlinear Analysis, 47, 777-787, 2001.

[24] T. Nagai, T. Senba and K. Yoshida, Application of the Trudinger-Moser inequality to a parabolic system of chemotaxis, Funk. Ekv., 40, 411-433, 1997.

[25] E. Onofri, On the positivity of the effective action in a theory of random surfaces, Commun. Math. Phys., 86, 321-326, 1982.

[26] C.S. Patlak, Random walk with persistence and external bias, Bull. Math. Biophys., 15, 1953, 311-338.

[27] B. Perthame, Frontiers in Mathematics, Transport Equation in Biology, Birkhäuser, 2007.

[28] C.J. Weijer, Dictyostelium morphogenesis, Curr. Opin. Genet. Dev., 14, 392-398, 2004. 